\title{
Variational analysis of drifter positions and model outputs for the reconstruction of surface currents in the central Adriatic during fall 2002
}

\begin{abstract}
V. Taillandier, ${ }^{1,2}$ A. Griffa, ${ }^{1,3}$ P. M. Poulain, ${ }^{4}$ R. Signell, ${ }^{5}$ J. Chiggiato, ${ }^{6,7}$ and S. Carniel ${ }^{7}$
Received 7 February 2007; revised 13 September 2007; accepted 7 December 2007; published 4 April 2008.

[1] In this paper we present an application of a variational method for the reconstruction of the velocity field in a coastal flow in the central Adriatic Sea, using in situ data from surface drifters and outputs from the ROMS circulation model. The variational approach, previously developed and tested for mesoscale open ocean flows, has been improved and adapted to account for inhomogeneities on boundary current dynamics over complex bathymetry and coastline and for weak Lagrangian persistence in coastal flows. The velocity reconstruction is performed using nine drifter trajectories over $45 \mathrm{~d}$, and a hierarchy of indirect tests is introduced to evaluate the results as the real ocean state is not known. For internal consistency and impact of the analysis, three diagnostics characterizing the particle prediction and transport, in terms of residence times in various zones and export rates from the boundary current toward the interior, show that the reconstruction is quite effective. A qualitative comparison with sea color data from the MODIS satellite images shows that the reconstruction significantly improves the description of the boundary current with respect to the ROMS model first guess, capturing its main features and its exchanges with the interior when sampled by the drifters.
\end{abstract}

Citation: Taillandier, V., A. Griffa, P. M. Poulain, R. Signell, J. Chiggiato, and S. Carniel (2008), Variational analysis of drifter positions and model outputs for the reconstruction of surface currents in the central Adriatic during fall 2002, J. Geophys. Res., 113, C04004, doi:10.1029/2007JC004148.

\section{Introduction}

[2] Lagrangian data in Near Real Time (NRT) are now available over most of the world ocean and in many regional and coastal areas thanks to a number of programs including subsurface floats and surface drifters. This surge in Lagrangian data has motivated in the last few years the development of appropriate methods to use the data in an optimal way in conjunction with results from Ocean General Circulation Models (OGCMs) to improve the description and prediction of the velocity field $\mathbf{u}$. Lagrangian instruments are floating buoys that follow the ocean current in good approximation, providing information on their position $\mathbf{r}$ at time intervals $\Delta t$ of the order of minutes to days depending on the applications. The positions $\mathbf{r}$ are related to the drift and therefore to the velocity field, and the challenge is to find appropriate ways to extract the $\mathbf{u}$ information from $\mathbf{r}$.

\footnotetext{
${ }^{1}$ RSAOS-MPO, Miami, Florida, USA.

${ }^{2}$ Now at LOCEAN, Paris, France.

${ }^{3}$ CNR-ISMAR, La Spezia, Italy.

${ }^{4}$ INOGS, Trieste, Italy.

${ }^{5}$ U.S. Geological Survey, Woods Hole, Massachusetts, USA.

${ }^{6}$ ARPA-SIM Emilia Romagna, Bologna, Italy.

${ }^{7}$ CNR-ISMAR, Venice, Italy.
}

Copyright 2008 by the American Geophysical Union. 0148-0227/08/2007JC004148\$09.00
[3] The simplest approach is to consider $\Delta \mathbf{r} / \Delta \mathrm{t}$ as a proxy of the Eulerian velocity $\mathbf{u}$ and to use it directly to correct $\mathbf{u}$ from the OGCM outputs [e.g., Hernandez et al., 1995; Ishikawa et al., 1996]. This simple approach is limited since it holds only when $\Delta t$ is significantly smaller than the Lagrangian timescale $T_{L}, \Delta t \ll T_{L}$ (where $T_{L}$ is typically of the order of $1-5 \mathrm{~d}$ in the ocean surface and $5-15 \mathrm{~d}$ in subsurface). A more general and powerful approach, valid also for $\Delta \mathrm{t} \sim \mathrm{T}_{\mathrm{L}}$ is to take into account the Lagrangian nature of the data introducing an appropriate observational operator based on the particle advection equation. The velocity correction $\Delta \mathbf{u}$ is then performed minimizing the difference between observed positions $\mathbf{r}$ and model forecasted positions from numerical trajectories. A number of different methods taking into account the Lagrangian nature of the data have been proposed in the literature and applied to either reconstructing the velocity field at a given time [Taillandier et al., 2006a], or to assimilating the information in OGCMs. The proposed assimilation methods range from Optimal Interpolation (OI) methods [Molcard et al., 2003, 2005], to Kalman filtering [Ide et al., 2002; Kuznetsov et al., 2003; Salman et al., 2006] and variational methods [Kamachi and O'Brien, 1995; Taillandier and Griffa, 2006; Nodet, 2006]. These methods have been tested in a number of idealized models and recently applied to in situ Argo floats using a realistic OGCM [Taillandier et al., 2006b]. The results are very encouraging, indicating the potential of using Lagrangian data for velocity correction. 
[4] The applications performed until now have been targeted mostly to open ocean flows, at large scales or regional scales. Applications in coastal environments are expected to pose additional challenges, related to the high inhomogeneity and to the presence of a number of competing scales of motion. This extended spectrum of scales of motion does not allow any clear separation between Lagrangian scales (representative of trajectory observations) and Eulerian scales (representative of flow simulations), and raises multiscale analysis issues. In this paper we present a study where Lagrangian data from drifters are used together with results from an OGCM to reconstruct the surface velocity field $\mathbf{u}$ in a coastal region with high inhomogeneity using a variational approach. The velocity reconstruction can be seen as a first step toward assimilation [Molcard et al., 2003, 2005; Taillandier et al., 2006a]. Once the model velocity is corrected at the level where the Lagrangian data are, in fact, the correction $\Delta \mathbf{u}$ can be assimilated in the model using appropriate methods that balance the velocity correction in terms of mass field correction. Also, the velocity reconstruction is useful per se, for instance, in order to improve particle prediction in the flow. This is a potentially important point since in many practical applications involving coastal flows, such as oil spill management or search and rescue, velocity outputs from operational models might be available while source codes might not be. This means that even though velocity data are expected to be fully exploited only by assimilating them in the OGCMs, in some cases this will not be possible, while it will always be possible to perform a correction of the velocity field blending model outputs and data.

[5] The velocity reconstruction is performed in a coastal area of the central Adriatic Sea, a subbasin of the Mediterranean Sea, using data from surface drifters and outputs from a state of the art model (ROMS) during the experiment DOLCEVITA in 2002. The area is especially challenging since it is characterized by a swift boundary current (Western Adriatic Current, WAC) flowing along the shelf with high shear and by an interior flow with high variability and intense mesoscale activity. The same region has also been studied in the framework of another recent experiment, the Dynamics of the Adriatic in Real Time DART06, where additional drifters have been launched in the boundary current and in the interior. Here we focus on the exchange between the WAC and the interior which is expected to play an important role in the ecology of the area, since the WAC advects the water of the Po River, rich in nutrients, therefore heavily influencing productivity. The WAC is subject to significant instability, especially in the considered area which is strongly topographically controlled and characterized by the presence of a cape (the Gargano Cape), leading to intrusions in the interior flow. We consider a period of $45 \mathrm{~d}$ between 1 October and 15 November 2002, when there is good drifter coverage of the WAC in the vicinity of the Gargano Cape, and we use them to correct the surface model velocity. Particular interest is given to quantities characterizing the rate of export from the boundary current and residence times in the area. A qualitative comparison between the reconstructed field and independent data from satellite color images is also performed.

[6] The reconstruction is based on a methodology that optimally blends model outputs and Lagrangian data, pre- viously developed by Taillandier et al. [2006a], and applied to flows in the open ocean. When applying the method, the first conceptual question is: what are the scales of motion that we want to address? For a coastal flow in midlatitude like the one considered here the scales of interest cover seasonal (or interannual), to mesoscale (order of a few days), down to submesoscale (order of $1 \mathrm{~d}$ or less), which include oscillations such as tides and inertial and turbulent submesoscale processes. In the central Adriatic, tides are not dominant but the inertial signal is significant, especially for surface summer flows. Ideally, OGCMs like the one considered here, with resolution of the order of few kilometers, could resolve almost all these processes, except for submesoscale turbulence which is expected to be only partially resolved. In practice, there are a number of issues in addition to space and time resolution that introduce additional model errors and further limit this capability. Model errors come from incomplete knowledge of forcing, smoothing of scales, as well as limitations in the resolved physics (for instance, OGCMs are usually hydrostatic and use parameterization to describe mixing processes). As a consequence, the details of the turbulent submesoscale flow cannot usually be described by the OGCMs even when resolution is of the order of $1 \mathrm{~km}$ or less, and often even inertial oscillations are not correctly reproduced since they depend on small-scale fluctuations of wind forcing, often not known in details. Regarding data resolution, surface drifters can provide high-frequency position data $(\Delta t$ of the order of half hour), in principle providing information in the submesoscale range, but in practice the drifter density is usually relatively low, so that at any given time, the data coverage is not sufficient to constrain the field. As a consequence of the limitations in both models and data, only the mesoscale component of the flow can be considered truly "resolved", and it is therefore the target of our reconstruction. With respect to the previous open ocean application [Taillandier et al., 2006a], here the presence of coastal inhomogeneity and boundaries is addressed and the influence of similar Eulerian and Lagrangian timescales is studied.

[7] The paper is organized as follows. In section 2, the Adriatic region and the period of interest are described, while the drifter data and model results used in the reconstruction are introduced in section 3. The methodology is discussed in section 4 and the details of its application and the considered diagnostics are given in section 5. Results are shown in section 6 and a summary, and discussion is provided in section 7 .

\section{Area and Period of Interest}

[8] The area of interest is the coastal region around the Gargano Cape along the Italian coast, in the central western Adriatic Sea (Figure 1). The topography is characterized by a well-defined shelf extending up to the $150 \mathrm{~m}$ isobath and widening south of the cape, and by a sill, the Palagruza Sill, extending offshore the Cape toward the eastern coast and separating two deep depressions, the South Adriatic Pit (SAP) and the Jabuka or Middle Adriatic Pit (MAP).

[9] The circulation in the area is part of the general cyclonic circulation of the Adriatic [Poulain, 2001], forced by buoyancy input and wind. Analysis of historical drifter 


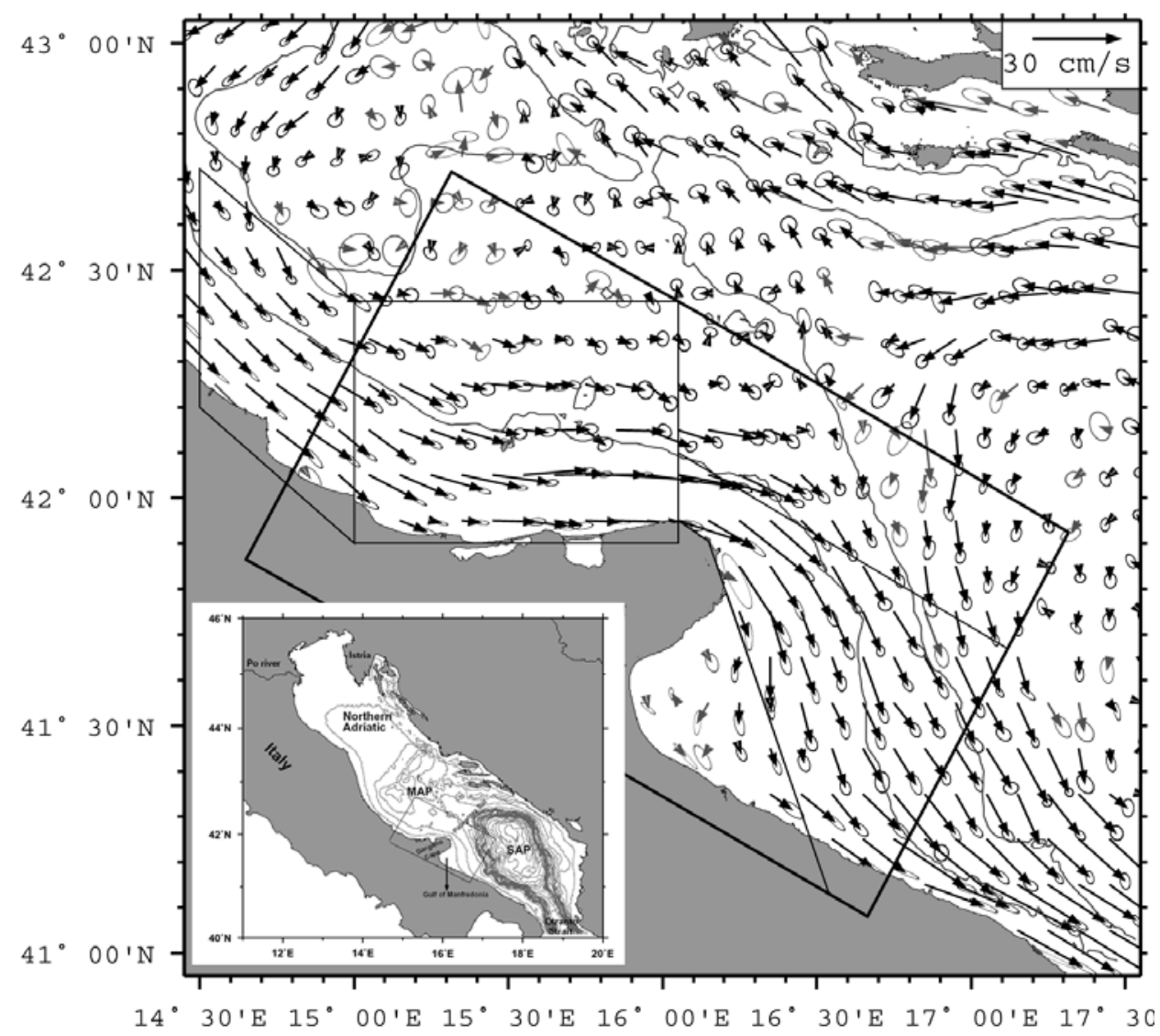

Figure 1. Mean flow after Veneziani et al. [2007], obtained by averaging the historical drifter velocities over 0.1 degree square bins. The standard error ellipses are computed with respect to the major and minor axis of variability. Also shown are the 100 and $160 \mathrm{~m}$ isobaths. The topography of the Adriatic Sea and the location of the area of interest are shown in the inset.

data in the area [Veneziani et al., 2007] shows a welldefined boundary current, the WAC, flowing to the southeast on the shelf and extending up to the $150 \mathrm{~m}$ isobath, with a core centered on the $100 \mathrm{~m}$ isobath, and widening downstream the cape (reported in Figure 1 of Veneziani et al. [2007]). In the mean flow, the WAC appears connected with the interior flow through two cross-basin branches that are part of two cyclonic recirculations around the two depressions of the SAP and MAP, respectively. The mean flow on the sill between the two recirculations shows a clearly marked saddle (or hyperbolic) point (see also the circulation maps of Poulain [2001]). The WAC is energetic and with significant fluctuations especially close to the tip of the Gargano Cape, while the flow in the lee of the cape (Gulf of Manfredonia) is characterized by a weak recirculation with reduced drifter sampling suggesting that the region is usually isolated from the main current.

[10] The fate of particles in the area has also been studied by Veneziani et al. [2007] considering maps of historical drifter concentrations as a function of drifter initial conditions. It is found that the majority of drifters initialized in the WAC upstream the Cape tends to remain inside the boundary current, especially if located inside the $100 \mathrm{~m}$ isobath core. The number of particle escapes from the WAC shows a clear seasonal dependence, with higher values during fall and winter with respect to summer, and with drifters penetrating the interior following the northern crossbasin recirculation branch or leaving at the tip of the Cape, in the region of high variability connected with fluctuations of the "hyperbolic point" region. In particular, the percentage of drifters exiting the WAC is the highest in fall, reaching $35 \%$ of the total historical drifters released upstream the Cape.

[11] Qualitatively similar results have also been found by Ursella et al. [2006] analyzing historical drifter data in the northern and central Adriatic and by Bignami et al. [2007] considering satellite SeaWiFS images over the whole Adriatic Sea. The results suggest that exchanges between the boundary current and the interior occur in fall and winter, when the current is stronger and more developed. This in turns is related to the occurrence of significant buoyancy forcing, given mostly by the Po River discharge, and wind forcing dominated by the strong northeasterly Bora wind.

[12] The period considered in this study covers $45 \mathrm{~d}$ during fall 2002, from 1 October to 15 November. The wind forcing has been quite variable during this period, as shown in Figure 2a. Wind data come from operational forecasts of the Limited Area Model Italy (LAMI), nonhydrostatic numerical weather prediction model based on the model LM [Steppeler et al., 2003], with $7 \mathrm{~km}$ horizontal resolution and 3-hourly outputs. The wind index presented in Figure 2a has been computed averaging the LAMI winds 


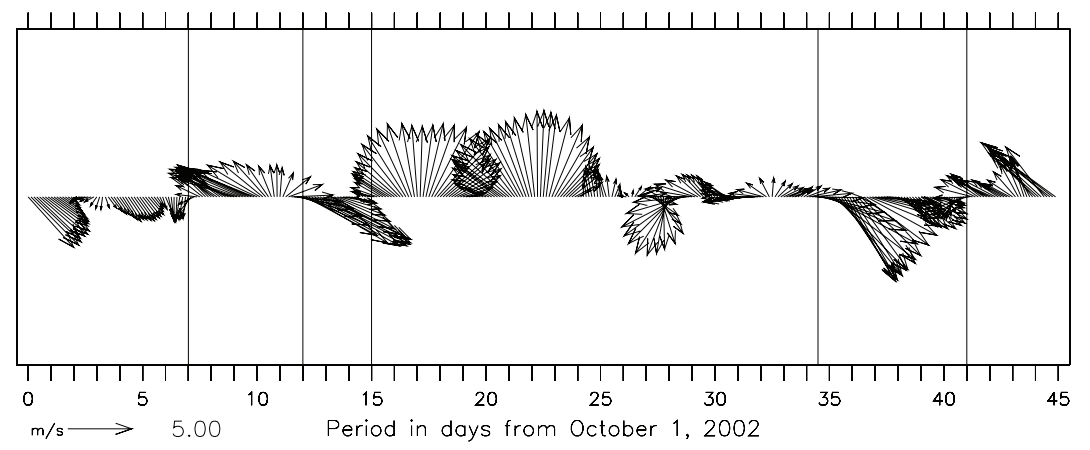

(a)

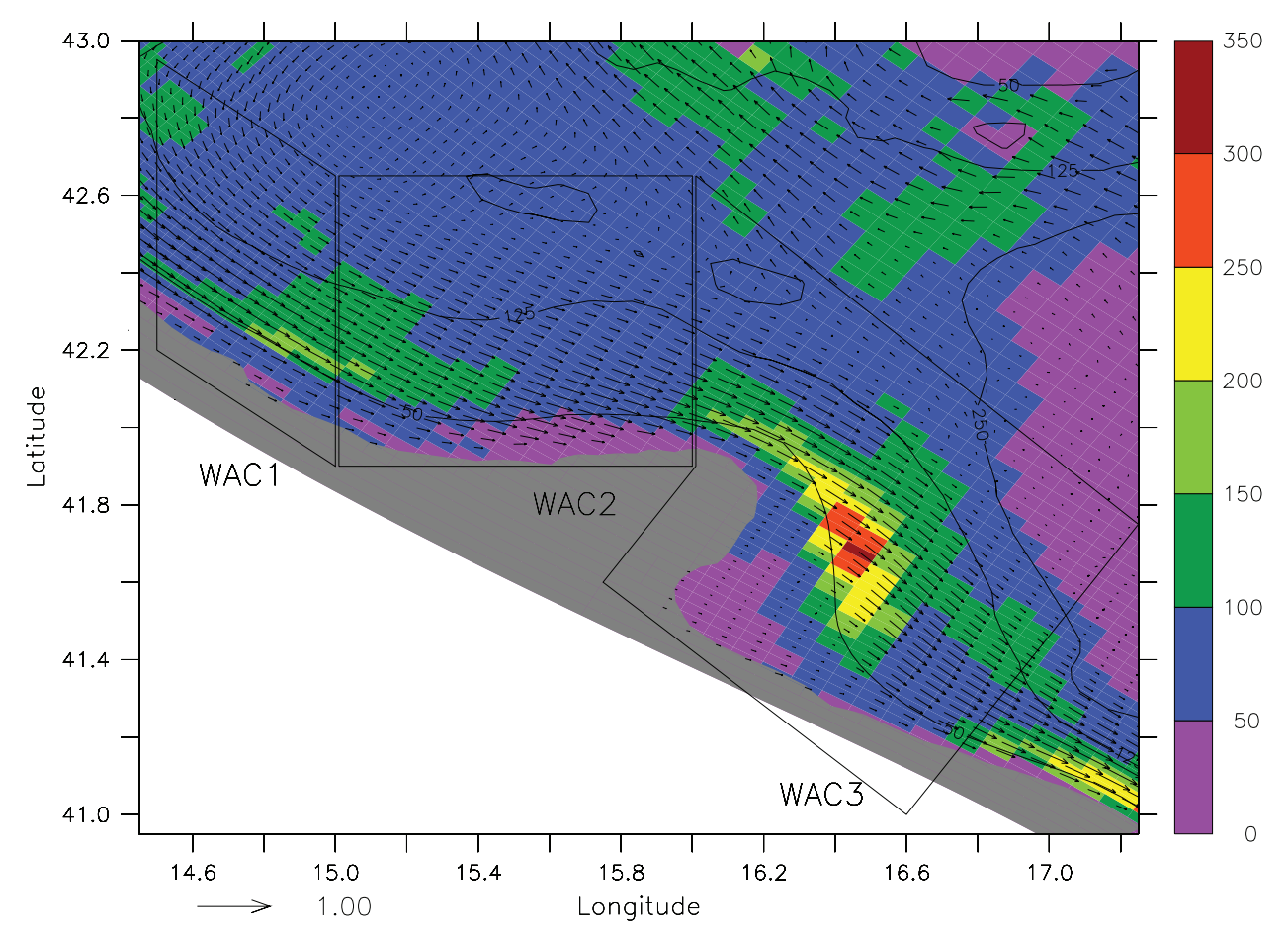

(b)

Figure 2. (a) Wind index from the LAMI forcing fields averaged over the three WAC partitions. The successive wind regimes, southward Bora-Mistral and northward Sirocco, are separated by straight lines. (b) Mean surface current (in $\mathrm{m} / \mathrm{s}$ ) and eddy kinetic energy (color bar in $\left.(\mathrm{cm} / \mathrm{s})^{2}\right)$, computed from the low-pass-filtered velocity fields of the ROMS model, over the whole period (from day 1 until day 45). The Western Adriatic Current is partitioned into three zones WAC1, WAC2, and WAC3; isobaths $50 \mathrm{~m}$, $125 \mathrm{~m}$, and $250 \mathrm{~m}$ are indicated by black lines.

over the area of interest, and preliminary tests made changing and widening this computation area show that it is a robust estimate of wind variability. Successive wind regimes can be seen in Figure 2a, with three wind regimes blowing southward (namely the Bora from NE and the Mistral from NW), alternated with three wind regimes blowing northward (namely Sirocco). To several quick bursts succeed longer but weaker wind episodes.

\section{Model and Data}

\subsection{The OGCM}

[13] The OGCM used in this study is version 2.2 of the Regional Ocean Modeling System (ROMS), a primitive equation, hydrostatic, finite difference, free surface model with the general kernel described by Shchepetkin and McWilliams [2005]. The horizontal resolution of the Adriatic implementation is variable (some $5 \mathrm{~km}$ in the area of interest), while 20 nonlinear terrain following s coordinate levels are used in the vertical. The model had been spun up with a 5-d diagnostic run using in situ data collected overall the Adriatic during September 2002 and optimally interpolated on the model grid. Advection for tracers is discretized using a MPDATA scheme [Margolin and Smolarkiewicz, 1998]. A weak grid-size-dependent, harmonic form (Laplacian), horizontal diffusivity is applied, while no horizontal viscosity is added. The pressure gradient term has been discretized by means of a density 
Jacobian with cubic polynomial fits [see Shchepetkin and McWilliams, 2003]. Parameterization of vertical mixing follows the generic length scale approach following Warner et al. [2005]. Surface forcing is provided by LAMI, with 3-hourly update. In particular, the net shortwave radiation is directly coming from LAMI, while the other fluxes are calculated interactively by ROMS using its own sea surface temperature and LAMI atmospheric data using BerliandBerliand formula [Budyko, 1974] for the net long-wave radiation and Fairall et al. [2003] COARE algorithms for turbulent heat and momentum fluxes. The model has an open boundary to the south where radiation boundary conditions are used for momentum and tracers, with superimposed the four major tidal harmonics (S2, M2, O1, K1) provided by Cushman-Roisin and Naimie [2002]. Fortyeight rivers (and springs) are included as well, as source of mass and momentum, using available daily discharges (Po, Pescara, Biferno Rivers) and monthly climatological values otherwise (following Raicich [1996]). Daily measured river temperature is specified for the case of the Po River.

[14] Here we consider a simulation of $45 \mathrm{~d}$ during the period of interest. Since the model reconstruction is performed off-line, instantaneous surface velocity outputs are extracted from the upper model layer, and stored every $3 \mathrm{~h}$ for a total of 360 fields. In the following, we briefly show the main statistics computed from these outputs in terms of mean flow and Eddy Kinetic Energy (EKE). Here the EKE corresponds to the temporal variability of the currents over $45 \mathrm{~d}$ on a spatial scale equal to the model mesh size. In order to better characterize the field, also for the diagnostics introduced in the following sections, we further subdivide the WAC region in three regions (represented in Figure 2b), as by Veneziani et al. [2007]. WAC1 and WAC2 are situated upstream the Gargano Cape, while WAC3 is downstream.

[15] In the mean surface circulation (arrows in Figure 2b), the WAC is represented by a laminar flow extending between the coast and approximately the isobath of $125 \mathrm{~m}$ with a core of intensity up to $50 \mathrm{~cm} / \mathrm{s}$. Upstream the Gargano Cape, in WAC1 and WAC2, the current appears guided by the coast line, while downstream it stays offshore the isobath of $50 \mathrm{~m}$ and does not enter inside the shallow shelf at the lee of the cape. The EKE (colors in Figure 2b) has been computed from low-pass-filtered velocity fields, with a cutoff period of $36 \mathrm{~h}$, since we are primarily concerned with the slow varying activity. High-frequency signals, mainly due to inertial oscillations (of period $19 \mathrm{~h}$ at these latitudes) generated by the successive wind bursts, are not taken into account. In the EKE map, the main fluctuations of the WAC occur downstream the tip of the cape, indicating recirculation cells along its inshore edge and branches of detrainment along its offshore edge. They suggest an important eddy activity, constrained by bathymetry, which could generate transient filaments extending offshore from the WAC toward the central basin or over the shallow shelf. Upstream the cape, the current fluctuations show a relative maximum along the isobath $50 \mathrm{~m}$ inside WAC1, while downstream a maximum can be seen south of WAC3, where the current impinges to the coast. These results, both for the mean and for EKE, appear consistent and qualitatively similar to those of Poulain [2001] and Veneziani et al. [2007] (see, for instance, Figure 1).

\subsection{Set of Drifter Position Data}

[16] The position data of nine surface drifters sampling the study area in October and November 2002 are considered. These drifters were all deployed in the northern Adriatic in late September 2002 during the Nato Undersea Research Centre (NURC) ADRIA02 trial. They are CODE designs [Poulain, 2001; Ursella et al., 2006] that measure the currents in the first meter of water with $1-2 \mathrm{~cm} / \mathrm{s}$ accuracy. All drifters were tracked with the Argos system on the NOAA polar-orbiting satellites. The Argos tracking has an accuracy of 300-1000 m and positions are typically available 6-12 per day. Four units were fitted with Global Positioning System (GPS) receivers to obtain hourly positions with better accuracy (about $10 \mathrm{~m}$ [see Barbanti et al., 2005]). Both Argos and GPS data were quality controlled and interpolated at $0.5 \mathrm{~h}$ uniform intervals using a kriging technique [Ursella et al., 2006]. They were low-passfiltered with Hamming filter with cutoff period of $36 \mathrm{~h}$, to remove the tidal and inertial components, and subsampled every $6 \mathrm{~h}$.

[17] The temporal distribution of the drifter data is represented in Figure 3a, during the $45 \mathrm{~d}$ period according to their location among the three WAC partitions. All the drifters cross these zones, successively, with residence times varying from a day to a week. However, their behavior is variable downstream the cape, in WAC3: two drifters leave the current (defined as within the isobath $125 \mathrm{~m}$ ) toward offshore whereas two others end up close to the shallow shelf.

[18] Individual trajectories are represented in Figures $3 b-3 e$, grouped as function of the wind regime they experience when they enter WAC1. Figure $3 \mathrm{~b}$ shows the first set of three trajectories entering WAC1 during the first southward wind regime. During the successive Sirocco regime, a large fluctuation of the coastal current appears in WAC2, as documented by the kinks in the three trajectories. These drifters pass the cape at the inshore edge of the current, and they experience a recirculation feature inside WAC3. Represented in Figure 3c, the second group of two drifters enters WAC1 during the first Sirocco regime, experiencing only one initial fluctuation during the complete pathway along isobath $50 \mathrm{~m}$. The other 4 drifters represented in Figures $3 \mathrm{~d}$ and 3 e enter inside WAC1 during the second Sirocco regime, characterized by two bursts followed by a calm.

[19] As it can be seen, there is no obvious relation between the wind regime and the fate of particles, even though the Sirocco wind seems to generate more fluctuations, especially if its onset follows a strong southward wind episode. In other words, the Bora and mistral might act as important preconditioning since they reinforce the WAC, while Sirocco episodes might trigger instabilities possibly related to the offshore Ekman transport at the surface.

\section{Methodology}

[20] A combined analysis of Lagrangian data (described in section 3.2) and velocity fields from OGCM outputs (described in section 3.1) can be performed by the variational approach set up by Taillandier et al. [2006a]. Some relevant aspects of this methodology are provided in section 4.1. The basic idea is to improve the realism of the simulated coastal flows which potentially contain phase 


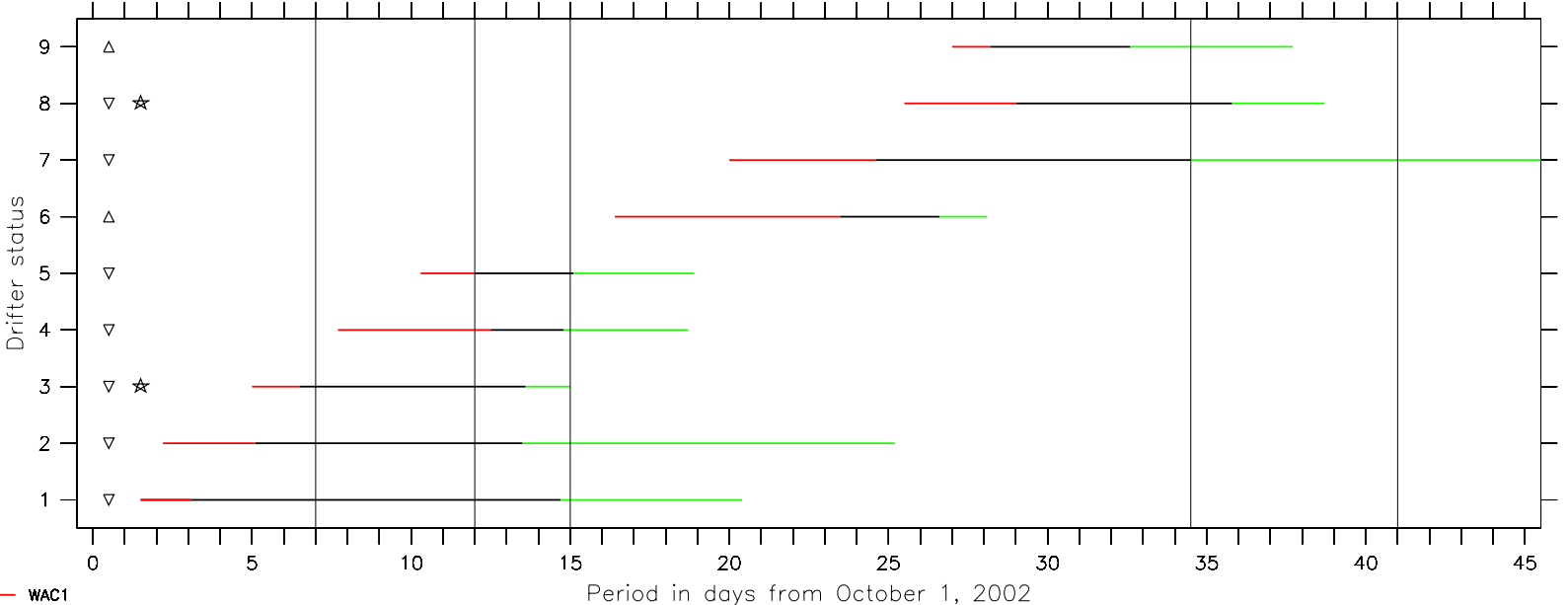

(a)
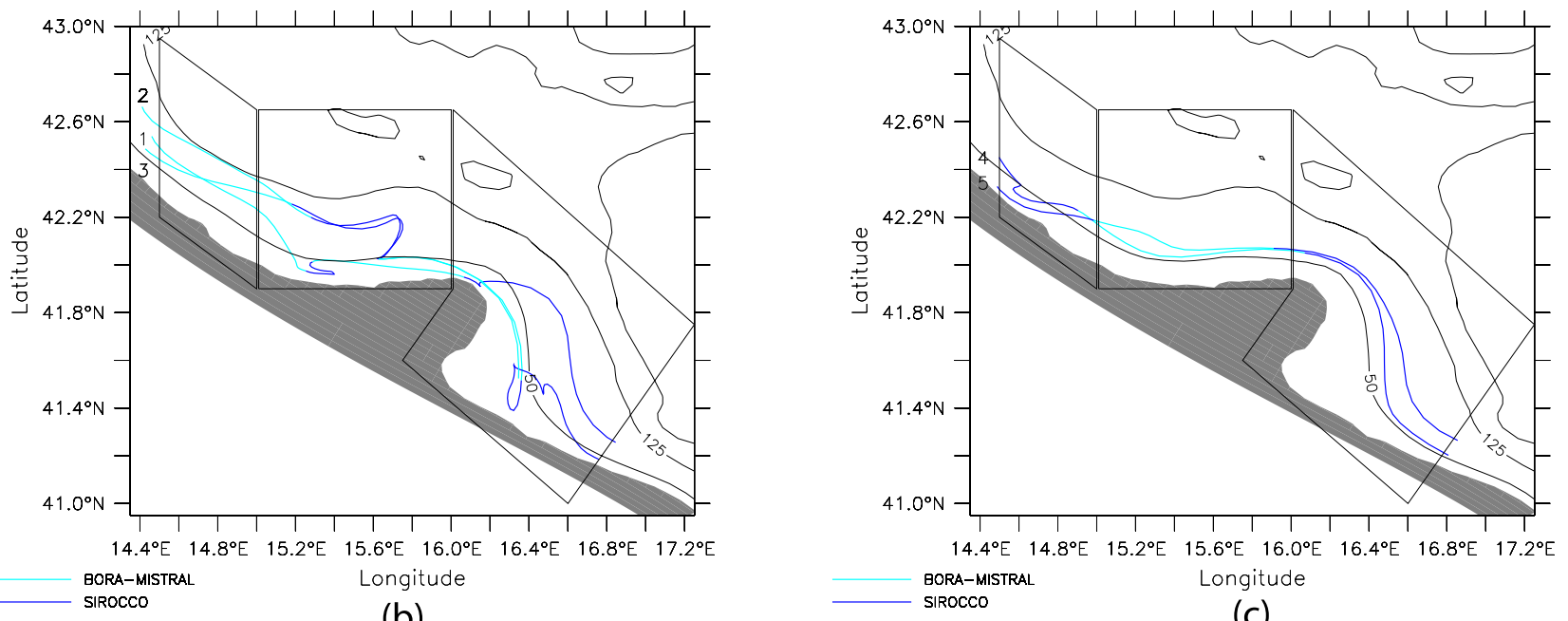

(b)
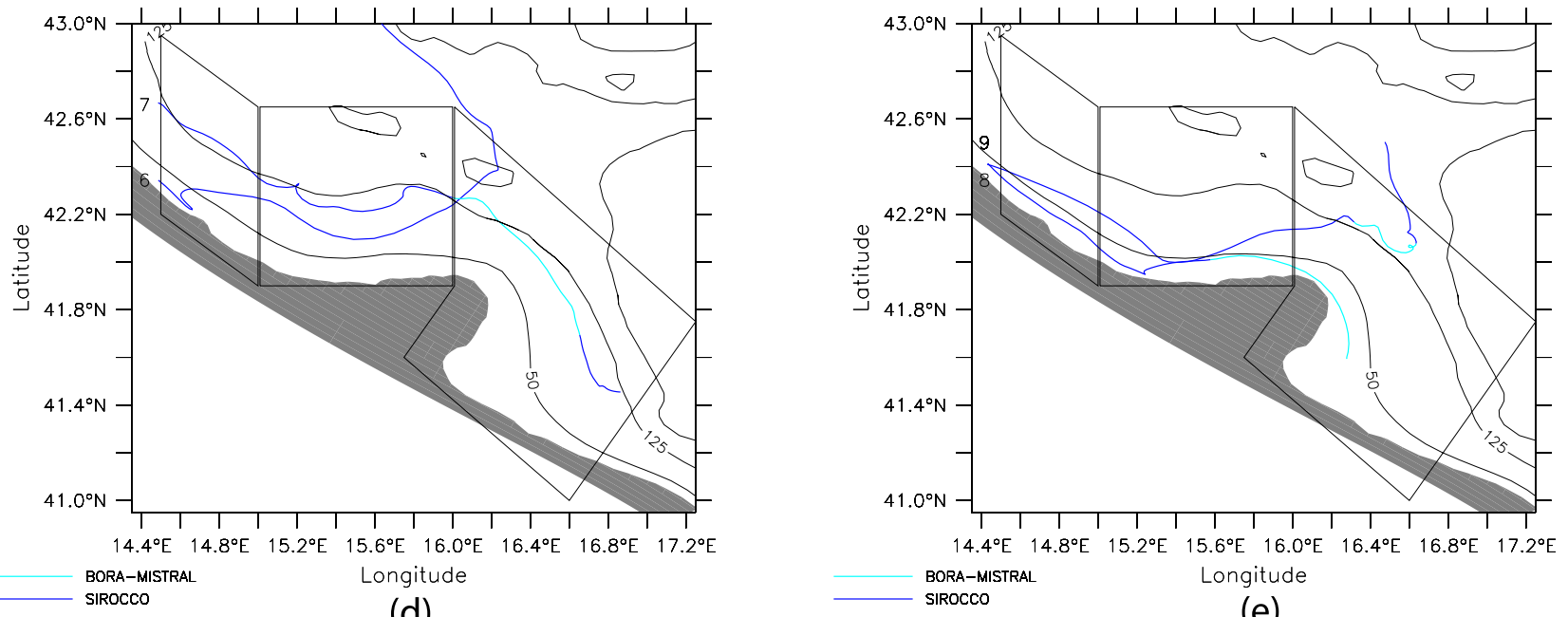

Figure 3. (a) Distribution of the position data set during the whole period. Horizontal and vertical axes indicate time in days and drifter identification number, and the colors indicate permanence in a given subregion. The status downstream of the cape is indicated for each drifter (inverted triangle if stays in WAC, triangle if exits from WAC, asterisk if dies). (b) Representation of the low-pass-filtered trajectories 1, 2, and 3. Wind regimes, reported from Figure 2a, are also indicated. (c) Same as Figure $3 \mathrm{~b}$ for trajectories 4 and 5. (d) Same as Figure $3 b$ for trajectories 6 and 7. (e) Same as Figure $3 b$ for trajectories 8 and 9. 

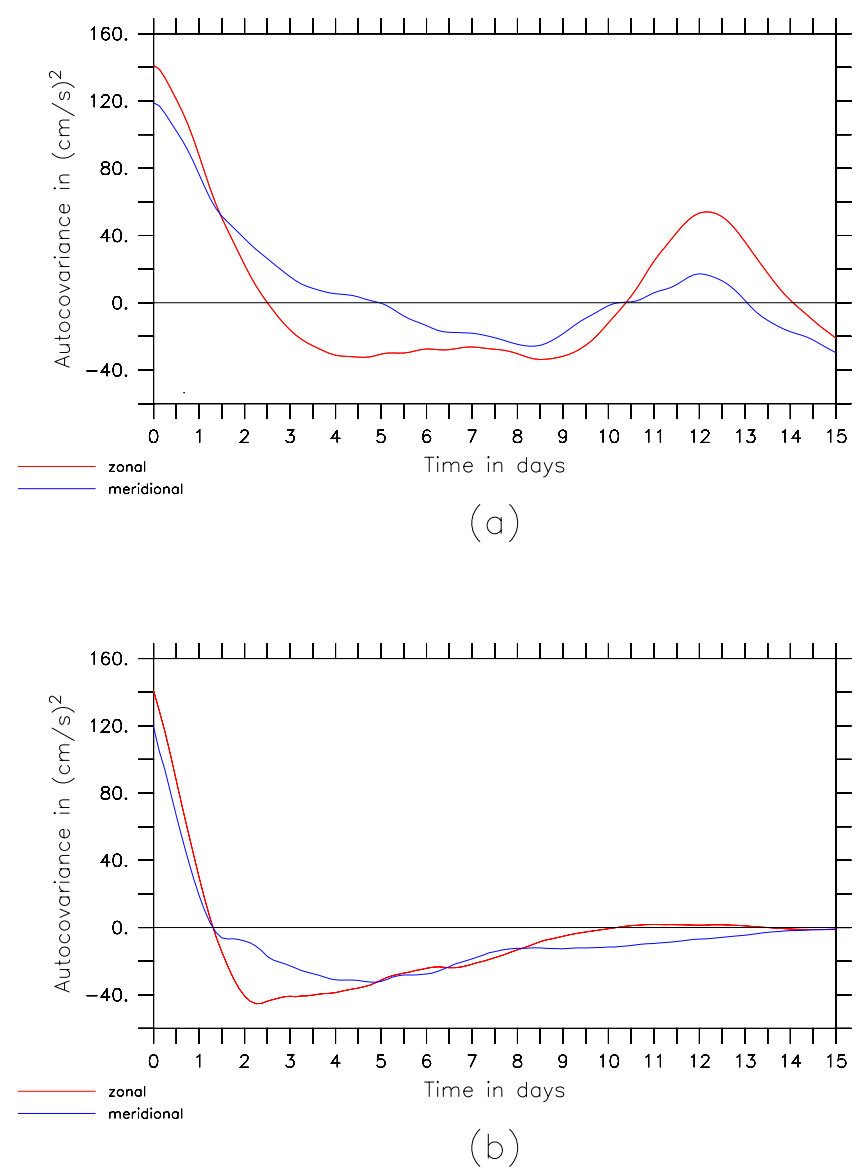

Figure 4. (a) Autocorrelation functions of Eulerian velocities. These velocity time series are provided by the numerical surface circulation averaged over the three WAC partitions and are low-pass-filtered. (b) Same as Figure 4a for Lagrangian velocities.

errors, i.e., velocity structures shifted in space and time that would generate point to point misfits between model and data. Considering observed drifter tracks, the local velocity field following each drifter path is to be modified within some correlation scales (R, T), which values are discussed in section 4.2 , in order to correct the phase errors occurring at these scales. Notice that the assimilation procedure is performed offline. This avoids any propagation by the model dynamics of velocity shift corrections, or any adjustment of other model errors (directly linked to boundary conditions, forcing or parameterization).

\subsection{A Variational Approach}

[21] The methodology consists in providing sequential velocity estimations with a fix spatial scale $R$ around simulated trajectories. For each sequence $\left[\mathrm{t}_{\mathrm{o}}, \mathrm{t}_{\mathrm{o}}+\tau\right]$, a time-independent correction $\Delta \mathbf{u}$ is added to the timedependent velocity field $\mathbf{u}(\mathrm{t})$ to provide an estimation $\mathbf{u}^{\text {est }}(\mathrm{t})$ over a characteristic timescale $\mathrm{T}$, as

$$
\mathbf{u}^{\text {est }}(\mathrm{t})=\mathbf{u}(\mathrm{t})+\Delta \mathbf{u}, \mathrm{t} \in\left[\mathrm{t}_{\mathrm{o}}-1 / 2(\mathrm{~T}-\tau), \mathrm{t}_{\mathrm{o}}+1 / 2(\mathrm{~T}+\tau)\right]
$$

[22] This velocity estimation is the one that minimizes the distance between trajectories simulated inside $\mathbf{u}$, and observed positions $\mathbf{r}^{\text {obs }}$. For each sequence $\left[t_{0}, t_{0}+\tau\right]$, this prediction misfit is expressed in the least squares sense by the cost function

$$
\mathrm{J}={ }^{1} / 2\left(\mathbf{r}^{\mathrm{obs}}\left(\mathrm{t}_{\mathrm{o}}+\tau\right)-\mathbf{H}_{\mathrm{NL}}(\mathbf{u})\right)^{\mathrm{T}} \cdot\left(\mathbf{r}^{\mathrm{obs}}\left(\mathrm{t}_{\mathrm{o}}+\tau\right)-\mathbf{H}_{\mathrm{NL}}(\mathbf{u})\right)
$$

where superscript $\mathrm{T}$ denotes the vector transpose, whose components are assumed independent and associated to Gaussian homogeneous errors. The observational operator $\mathbf{H}_{\mathrm{NL}}(\mathbf{u})$ provides position prediction at time $\mathrm{t}_{\mathrm{o}}+\tau$ using trajectory simulations by the nonlinear equation

$$
\mathrm{d}_{\mathrm{t}} \mathbf{r}=\mathbf{u}(\mathbf{r}(\mathrm{t}), \mathrm{t}), \mathrm{t} \in\left[\mathrm{t}_{\mathrm{o}}, \mathrm{t}_{\mathrm{o}}+\tau\right] \text { with } \mathbf{r}\left(\mathrm{t}_{\mathrm{o}}\right)=\mathbf{r}^{\mathrm{obs}}\left(\mathrm{t}_{\mathrm{o}}\right)
$$

where $d_{t}$ is the first-order derivative in time. The minimization of $\mathrm{J}$ is performed by a steepest descent procedure along its gradient. Notice that the gradient expression, detailed by Taillandier et al. [2006a], includes a priori information on the length scale $\mathrm{R}$ of $\Delta \mathbf{u}$ inserted through characteristic background error correlations for $\mathbf{u}$.

\subsection{Implementation for Coastal Currents: Case of the WAC}

[23] The method presented in section 4.1 is now to be applied to the case of coastal currents such as the WAC. This requires specific parameterizations to be set up from information about spatial scales $\mathrm{R}$ and timescales $(\mathrm{T}, \tau)$ of the WAC fluctuations.

[24] Regarding timescales, two time series of eddy velocities averaged inside the three WAC partitions have been extracted from the numerical velocity fields and associated trajectory simulations (described in section 5). The corresponding autocorrelation functions are obtained after a low-pass-filtering of the two time series at a cutoff period of $36 \mathrm{~h}$ (see Figure 4). The timescales are identified with the persistence of the signals, in the order of $\mathrm{T}=2 \mathrm{~d}$ for Eulerian velocities (Figure 4a), and $\tau=1 \mathrm{~d}$ for Lagrangian velocities (Figure 4b).

[25] Regarding spatial scales, the case of the WAC involves frontal dynamics which settle horizontal barriers along the offshore edge of the coastal current and isolate velocity correlations of each side. So two distinct regimes can be considered separately, one inside the WAC and one outside the WAC. Hence, two distinct velocity corrections can be provided by the method, $\Delta \mathbf{u}^{\text {in }}$ inshore and $\Delta \mathbf{u}^{\text {off }}$ offshore. The velocity estimation, expressed in equation (5), is then refined to the relation

$$
\begin{gathered}
\mathbf{u}^{\text {est }}(\mathrm{t})=\mathbf{u}(\mathrm{t})+\alpha \cdot \Delta \mathbf{u}^{\text {in }}+(1-\alpha) \cdot \Delta \mathbf{u}^{\text {off }}, \\
\mathrm{t} \in\left[\mathrm{t}_{\mathrm{o}}-1 / 2(\mathrm{~T}-\tau), \mathrm{t}_{\mathrm{o}}+1 / 2(\mathrm{~T}+\tau)\right]
\end{gathered}
$$

where $\alpha$ is equal to 1 inside the WAC extension, and quickly tends to 0 toward the basin interior.

[26] Each velocity correction $\left(\Delta \mathbf{u}^{\text {in }}\right.$ or $\left.\Delta \mathbf{u}^{\text {off }}\right)$ is specified by its spatial correlation structure, associated to a specific motion scale (R, T), using background error correlations. In practice, this structure can be modeled by the solution of a diffusion equation over a length scale $\mathrm{R}$, which interprets the spatial autocovariance for the zonal or the meridional velocities [Derber and Rosati, 1989]. Moreover, such 
Laplacian grid point smoother allows to account for anisotropic correlations, inherent to the description of coastal flows, which are imposed by the presence of coastlines [Weaver and Courtier, 2001].

[27] So each selected motion scale, taken distinctly inside and outside the WAC, would be the only one corrected by the method. Considering equation (6), this overall estimation is superimposed to other motions scales which have been filtered out or which cannot be directly described by Lagrangian data. Several preliminary tests have provided the sensitivity of estimates with respect to the choice of the interface design separating the coastal and offshore zones, and with respect to the values for $\mathrm{R}$ in each side. The parameterization used in this study is the following.

[28] The first correction $\Delta \mathbf{u}^{\text {off }}$ is related to the dynamics outside the coastal current, in which motions at timescale $\mathrm{T}$ can be represented with a length scale $R^{\text {off }}=20 \mathrm{~km}$. Their correlation structure is modeled over the whole basin. The second correction $\Delta \mathbf{u}^{\text {in }}$ is related to the WAC dynamics, in which motions at timescale $\mathrm{T}$ are represented with a smallerscale $\mathrm{R}^{\text {in }}=10 \mathrm{~km}$. Their correlation structure is modeled over a reduced domain, bounded by the coastline and the edge of the coastal current. This offshore edge is identified with the isobath of $125 \mathrm{~m}$.

\subsection{Estimation Procedure}

[29] The method is applied performing an analysis of the trajectories during the successive daily sequences covering the $45 \mathrm{~d}$ period. The $\Delta \mathbf{u}$ correction is computed with respect to the mesoscale velocity field with timescale $\mathrm{T}=2 \mathrm{~d}$. In practice, this is done computing a "first guessed" velocity field averaging the OGCM output over $2 \mathrm{~d}$ and correcting it using information from the trajectories over the same period. This procedure is made every day, obtaining daily corrected estimates of the velocity. The high-frequency fluctuations are not corrected, and the ones of the first guessed flow are maintained.

[30] Notice that the velocity correction is not propagated by the model dynamics as the methodological step toward online assimilation is not implemented. Since the data coverage is not uniform over the $45 \mathrm{~d}$ period, we can expect time windows without estimation. In Figure $3 \mathrm{a}, \mathrm{WAC} 1$ is not covered during days $12-16$ and $29-45$, WAC2 is not covered during days $1-3,16-23$ and $36-45$, WAC3 is not covered during days $1-13$ and 26. Small gaps can still be compensated with the superimposition of earlier or latter velocity corrections, but a midperiod window of some days would remain without estimation.

[31] On the other hand, assuming that the method is implemented into an operational system, this data density would be sufficient to cover the period, as indicated by the assimilation performance of Argo float positions in open ocean configuration [Taillandier and Griffa, 2006]. Moreover, such online application has been performed without any significant increase of computer time.

\section{Diagnostics}

[32] The velocity field $\mathbf{u}$ has been reconstructed using the methodology in section 4 for the $45 \mathrm{~d}$ of interest, obtaining a time series of corrected velocities we refer to as "estimates." The question to be addressed at this point is how to evaluate these estimates. Taillandier et al. [2006a] tested the method using the "twin experiment" approach, where a specific numerical simulation is regarded as the "true ocean" and synthetic data are computed in it. The reconstruction is then performed using the synthetic data to correct the results of a different simulation, characterized by different initial conditions representing our incomplete knowledge of the ocean state. The method can be evaluated quantitatively since the truth is known, even though results might be overly optimistic given that the synthetic data are perfectly compatible with the OGCM. When using real in situ data, as in this paper, the truth is obviously not known since is given by the real ocean state, and evaluating the results is not straightforward.

[33] Here we propose a hierarchy of tests and diagnostics to investigate the properties of the reconstruction. The first three sets of diagnostics are aimed at verifying internal consistency and impact of the method, and they are based on comparisons between results from the estimates and results from the original velocity field without corrections, which we refer to as "first guessed" or "background" results. The fourth and last diagnostic is based on a qualitative comparison with independent data from satellite images.

[34] The first test on the reconstruction is based on the particle prediction diagnostic and it is targeted to verify internal consistency of the method. The reconstruction method corrects $\mathbf{u}$ requiring that the difference between observed and simulated trajectories is minimized. As a consequence, we expect that the particle prediction is improved using the estimate with respect to the background.

[35] This is quantified computing an error prediction $\varepsilon$ for both the estimate and the background, as the distance between numerical and observed drifters during a $48 \mathrm{~h}$ period. In practice, each observed position provides an initial condition for a single trajectory simulation inside the velocity fields covering the next $2 \mathrm{~d}$. This provides a set of realizations at each position (sampled every $\Delta t=3 h$ ) of the 9 observed drifters, over which an average quantity is computed. Roughly speaking, the prediction improvement in the estimate with respect to the first guess is expected to provide an assessment of the efficiency of the cost function (defined in equation (2)).

[36] The following two tests are aimed at quantifying the impact of the reconstruction in terms of residence times and export rates which characterize the transport in the region of interest. In order to compute these statistics, an extensive set of numerical trajectories have been computed in the estimated and in the first guessed velocities, respectively. The trajectories are simulated using the Runge-Kutta scheme with a time step equal to $\Delta \mathrm{t}=3 \mathrm{~h}$ and then lowpass-filtered at the cutoff period of $36 \mathrm{~h}$. In order to avoid landing, numerical drifter motions are limited to the only sea mesh points by removing every normal displacements at the coast. Particles are launched in batches every $\Delta t$, releasing them inside the WAC along three cross-shore sections taken at longitudes $14.5^{\circ} \mathrm{E}, 15^{\circ} \mathrm{E}, 16^{\circ} \mathrm{E}$ corresponding to the upstream borders of WAC1, WAC2, WAC3, respectively. Launching resolution along the sections is $\Delta \lambda=0.01^{\circ}$, so that the number of drifters released between the coast line and the isobath of $125 \mathrm{~m}$ is equal to 43 upstream WAC1, 38 upstream WAC2, and 36 upstream WAC3. An example of trajectory 

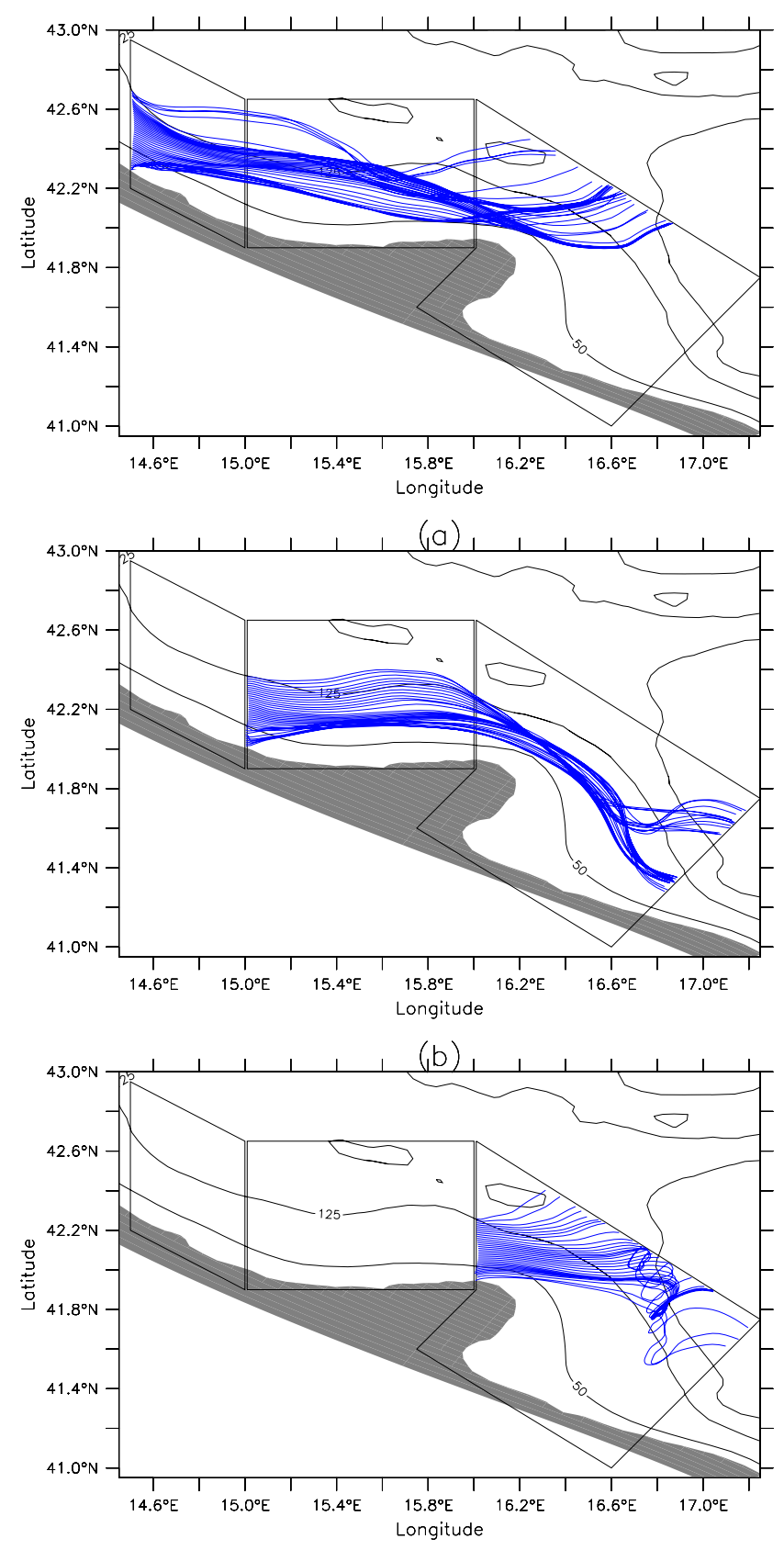

(c)

Figure 5. (a) Low-pass-filtered trajectories simulated inside the first guessed circulation, from the launching location upstream WAC1 at day $15(0000 \mathrm{~h})$. (b) Same as Figure 5a with a launching upstream WAC2. (c) Same as Figure 5a with a launching upstream WAC3.

simulation is given in Figure 5 for launchings along the three sections.

[37] These trajectories are then used to compute the diagnostics for each release, so that there is one estimate for each launching time, i.e., at each $\Delta \mathrm{t}=3 \mathrm{~h}$, and for each launching section. The estimates are computed over the "active" drifters defined as those that reach WAC3 and exit from it during the $45 \mathrm{~d}$ of integration, therefore excluding the "slow" particles whose fate is undefined, similarly to what was done by Veneziani et al. [2007] for the real drifters. For each release, the ratio between the active drifters and the ones initially released is computed and used as "quality control." The diagnostics are computed and considered significant only when this ratio is over $75 \%$. The residence time $\theta$ inside each region WAC1, WAC2 and WAC3 is computed as the average time spent by the active drifters in the region, while the export rate $\chi$ is given by the percentage of active drifters that do not exit from WAC3 across its southeastern boundary.

[38] Finally, the fourth test consists in a qualitative comparison of the first guessed and estimated fields with images provided by the Moderate Resolution Imaging Spectroradiometer (MODIS) onboard the Aqua satellite. The MODIS product we have chosen is level 2 chlor_a2, computed using the OC3M algorithm, which represents the near-surface chlorophyll a concentration but also includes significant contribution from colored dissolved organic matter, detritus degradation products and suspended sediments [see Bignami et al., 2007; Mauri et al., 2007]. These images provide information on the structure of the WAC, easily identifiable since characterized by more turbid and productive water of Po origin [e.g., Bignami et al., 2007]. In particular, we focus on the patterns of the edge of the WAC at a given time, and we compare it qualitatively with the velocity field and with the corresponding pattern of trajectories. The comparison is necessarily qualitative for a number of reasons. First of all we visualize a small set of trajectories, released at given sections and considered as arbitrarily "marked," while the features in the images originate from the evolution of the complete tracer field with unknown initial conditions. Also, and more fundamentally, our reconstruction is targeted to mesoscale flows (order of 1-2 d), while the images are instantaneous so that the small-scale events (of less than $1 \mathrm{~d}$ resolution), such as some plume structures or filaments, cannot be captured by the correction. Finally, the correction is based on drifters moving in the upper 1-2 $\mathrm{m}$ of the water column while the images capture surface effects which are expected to be directly influenced by the wind. Also, it should be noticed that the tracer depicted in the images is not passive and can be influenced by other phenomenon than advection only. For all these reasons the comparison is not focused on the detailed structure of the field, but rather on characterizing the main boundary current regimes, i.e., regimes with significant offshore transport versus regimes where the WAC is confined close to the coast and does not significantly exchange with the interior.

\section{Results}

\subsection{Particle Prediction Error}

[39] The time series of the particle prediction error $\varepsilon$ for time lags up to $48 \mathrm{~h}$ is shown in Figure 6 together with the RMS values for the background and the estimate. The background error and RMS grow almost linearly and $\varepsilon$ reaches approximately $30 \mathrm{~km}$ after $2 \mathrm{~d}$. This value appears quite high, but it is consistent with other values obtained in the same area with other OGCMs (A. Griffa, personal communication, 2006). The prediction of single particle trajectories is in fact a very hard test for a model, since particle trajectories are often chaotic [Aref, 1984] and highly 


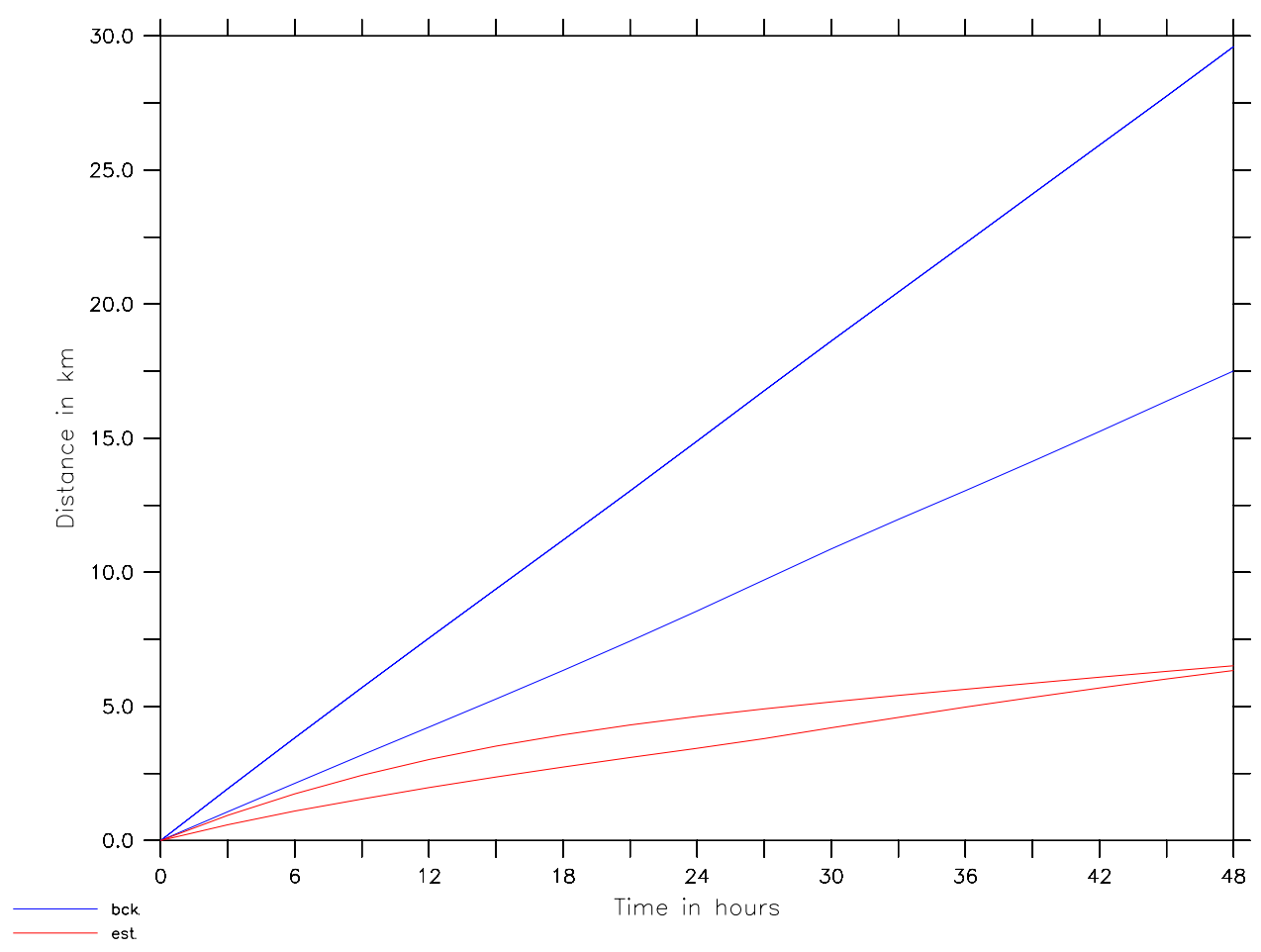

Figure 6. Distance between observed drifter positions and trajectories simulated inside first guessed (estimated) velocity fields on a $48 \mathrm{~h}$ forecast, in blue lines (red lines). Average (in solid lines) and standard deviation (in thin lines) are computed over the whole data set.

dependent on the initial conditions and on the details of the Eulerian velocity.

[40] The error $\varepsilon$ is significantly reduced in the case of the estimate, growing much more slowly and almost saturating around $7 \mathrm{~km}$. This is a clear indication that the estimated velocity field has been consistently corrected with respect to the trajectories. It shows also the major influence of the selected scales $(\mathrm{R}=10 \mathrm{~km}, \mathrm{~T}=2 \mathrm{~d})$ among the spectrum of the WAC dynamics in the prediction errors of drifter pathways.

\subsection{Residence Times}

[41] Examples of the residence time diagnostic $\theta$ are shown in Figure 7 as a function of the particle launching times for the background and the estimate. The three panels show values of $\theta$ computed for the three regions (WAC1, WAC2, and WAC3, respectively), considering particles launched upstream each region. The corresponding values of residence time for each of the 9 observed drifters are also shown in the plots.

[42] A general overview of the results shows significant differences between the background and the estimate, indicating that the reconstruction has a significant impact. Though $\theta$ maintains similar amplitude indicating that the numerical results are in the same range as the data. This is consistent with the fact that the model energetics (Figure 2b) is similar to the experimental one [see, e.g., Veneziani et al., 2007]. Notice that $\theta$ inside WAC2 or WAC3 is roughly twice as much as inside WAC1, because of the larger extension.

[43] Some particular events can be pointed out with respect to the shape of observed pathways. For example in WAC1 (Figure 7a), the motion of drifters 8 and 9 which cross this zone at days 26-29, tends to accentuate the estimated flow intensity, as shown also in Figures $11 \mathrm{~b}$ and 11c. So during this period, the estimated residence time is decreased (see Figure 7a). Another event that occurs earlier (days 17-24) shows a significant increase of the estimated residence time inside WAC1. This is due to the impact of drifter 7 along the offshore current edge which tends to reduce the estimated flow intensity. However, the quick reversal pathway of the drifter 6 along the coast (shown also in Figure 3e) has not this significant impact on the diagnostic.

[44] Considering the evolution of the residence time inside WAC2 (Figure 7b), the estimate provides a clear improvement over the background. This can be explained by an increase of eddy activity on the estimated circulation, which is weaker in the background circulation. In particular, the first drifters 1, 2, 3 provide an important contribution, with meandering features which accentuate the residence time for launching times before day 9. This can be seen, for instance, in Figure 8. In the same way, the slow motion of drifter 7 along the offshore current edge tends to increase the residence time reported at day 25 .

[45] Such comparisons are somehow difficult to lead inside WAC3 (Figure 7c), because the diagnostic is highly variable given the different current features and pathways. As a matter of fact, recirculation inside the shallow Gulf of Manfredonia are associated to high residence times; instead, a branch detrained offshore provides low residence times. So, only the most important misfits between background and estimated residence times can be qualitatively inter- 


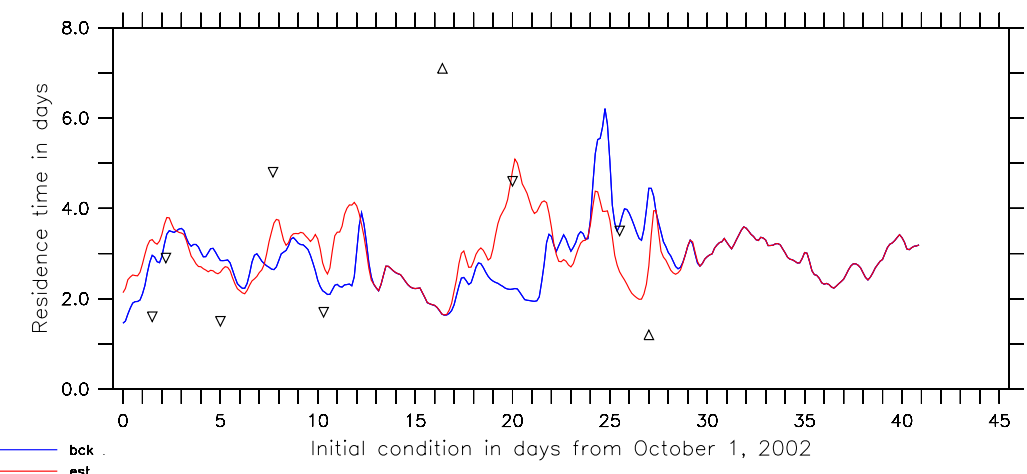

(a)

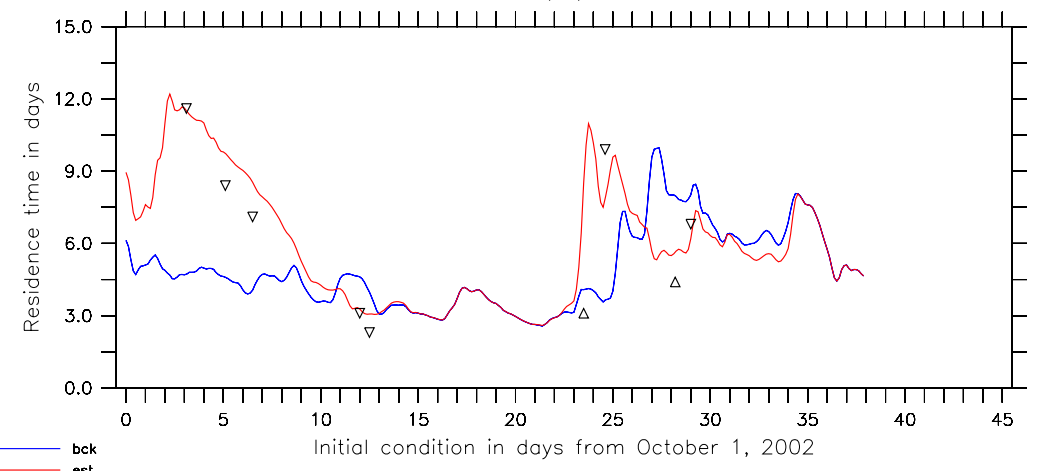

(b)

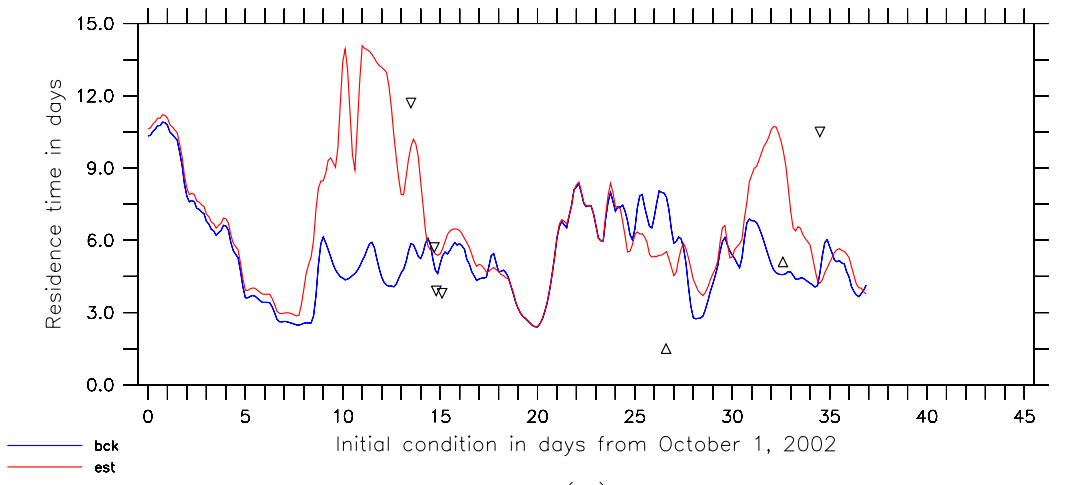

(c)

Figure 7. (a) Mean residence time, computed from trajectory simulations inside the first guessed velocity fields (blue lines) and the estimated velocity fields (red lines), or measured from real trajectories (inverted triangle if stays in WAC, triangle if exits from WAC). Plots are represented for residence times inside WAC1 with respect to a launching location upstream the same zone. (b) Same as Figure 7a inside WAC2. (c) Same as Figure 7c inside WAC3.

preted from Figure 7c, also in combination with the next diagnostic.

\subsection{Export Rates}

[46] As for the residence times, examples of the export rates $\chi$ for the background and the estimate are shown in Figure 9 for the three regions as a function of the particle launching times. The status of the 9 observed drifters are also shown in the plots, indicating whether they stay in the WAC or exit it.

[47] An overview of Figures 9a-9c shows marked differences between the background and the estimate, even more than in the case of $\theta$ (Figure 7). This is probably due to the fact that $\chi$ is a higher-order statistics, more strictly related to the details of particle pathways and therefore harder to reproduce with numerical modeling. Attenuation of partial or mixed cases can be seen in between the two trajectory fates (exported or trapped) downstream the cape (the closer to the cape, obviously the more deterministic trajectory fate). Furthermore, the diagnostic computed from the background velocity fields reveals a translation in time of peaks marking export events, according to the location of initial condition. It suggests for example that the peak at the launching day 23 upstream WAC1 (Figure 9a) marks the same export event than the peak at the launching day 25 upstream WAC2 (Figure 9b), or the peak at the launching day 29 upstream WAC3 (Figure 9c). Also evidenced in Figure 7 by slow varying residence times, the background 


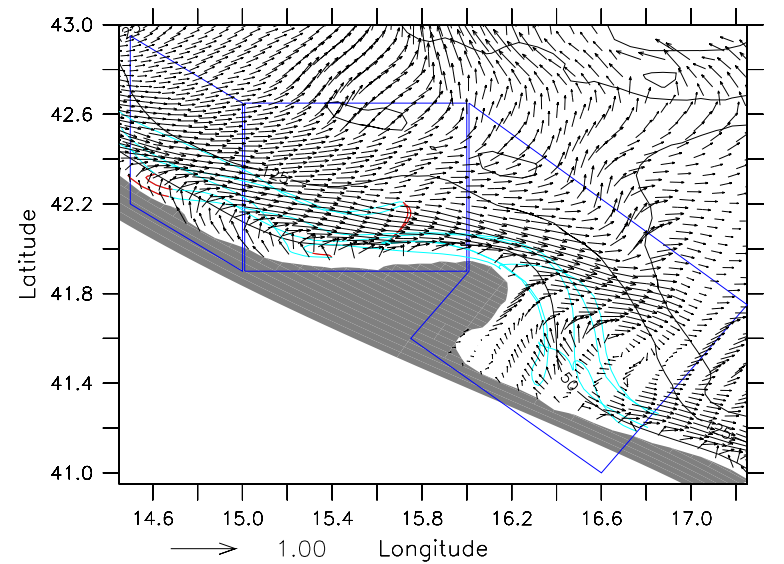

(a)

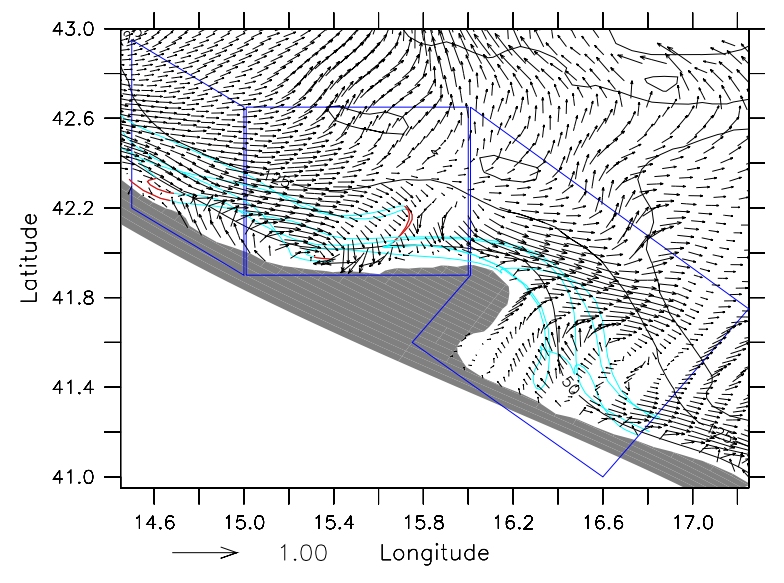

(b)

Figure 8. (a) Mean surface circulation over days $9-10$, given by the first guessed velocity fields. (b) Same as Figure 8a estimated velocity fields.

circulation provides well-structured transitions between the two trajectory fates. Instead, the data analysis infers episodic events on export rates, and it smoothes this quasilaminar behavior by introducing eddy activity in the estimated circulation.

[48] The pathways toward offshore have a reduced number of occurrences inside the estimated circulation. This is in general agreement not only with the fate of the 9 drifters considered here, but also with the results of Veneziani et al. [2007], showing that approximately $35 \%$ of drifters leave the current in autumn. In particular, the data analysis tends to disable any export pathways during the passage of the first four drifters $1,2,3$, 4. It clearly appears at the beginning of the period, see Figure 9 (days $1-13$ in WAC1, days $2-15$ in WAC2, days $9-17$ in WAC3). Notice that it can be related to an increase of the residence times $\theta$ inside WAC3 during the same time window, see Figure 7c. Apart from the time windows not covered by observations (described in section 4.3), there are sparse estimated export events as in Figure 8. Two episodes occur from WAC1 (Figure 9a), at days $15-18$ and day 27 in agreement with the fate of the drifters 6 and 9. Only one occurs from WAC2 and WAC3 (Figures 9b and 9c) at day 23 and day 26, respectively, in agreement with the fate of drifter 6 .

\subsection{Comparison With Satellite Image}

[49] The comparison is done using two MODIS images, corresponding to 16 and 27 October, respectively, and characterized by different regimes. The 27 October image (Figure 11a) shows significant fluctuations in the boundary current (marked in yellow) with plumes extending toward the interior flow and indicating offshore transport, while during 16 October the current appears more confined without clear exchanges with the interior (see Figure 10a). The choice of the days have been dictated by image availability (no clouds and clear signal for the boundary current) and concurrent drifter data availability in the area around the Gargano Cape where the signal is most pronounced so that a significant correction can be seen in the reconstruction.

[50] The 16 October image (Figure 10a) is relative to the beginning of the second Sirocco episode, occurring after a southward wind episode (Figure 2). Small-scale fluctuations can be seen at the edge of the current, but they do not appear to lead to offshore plumes or filaments, rather tending to roll toward the coast. The boundary current signal appears mostly confined along the coast in the area of the Gargano Cape. The superimposed trajectories follow the main pattern of the current flowing southward without showing the details of the small-scale structures. Only in the lee of the cape, in the Gulf of Manfredonia, the occurrence of a smallscale recirculation can be seen. The trajectories are also superimposed to the 2-d mean velocity from the background and estimate in Figures $10 \mathrm{~b}$ and 10c. The effect of the reconstruction appears to maintain the current closer to the coast along the cape, while inducing a small recirculation in the lee. In order to better visualize the effects of the correction, particle trajectories released along the WAC3 section the day earlier, i.e., 15 October, have been considered. Their advection pattern inside background and estimated circulations is represented in Figures $10 \mathrm{~d}$ and 10e, respectively. While the background trajectories (Figure 10d) show a large-scale meander carrying the trajectories offshore downstream the cape (a pattern that is not observed in Figure 10a), the estimate trajectories (Figure 10e) follow the WAC without being exported toward the interior and partially recirculating in the Gulf of Manfredonia, similar to that suggested in the image.

[51] The image of 27 October (Figure 11a) shows a different situation, with larger instabilities occurring in the WAC, and two clear patterns of export in WAC2 and WAC3 at the tip of the cape. This event occurs during the second and extended period dominated by Sirocco (Figure 2), during a brief intermediate episode of southward wind. Four drifters sample the zone, three upstream the cape in the boundary current and one showing a clear exit path from it. A comparison between the background and estimate velocity (Figures 11b and 11c) shows that the reconstruction creates an eddy structure upstream the cape and an offshore plume close to the tip. The pattern of the trajectories launched at the WAC3 section is significantly different for the background and the estimate (Figures 11d and 11e). Background trajectories stay inside the boundary current, 


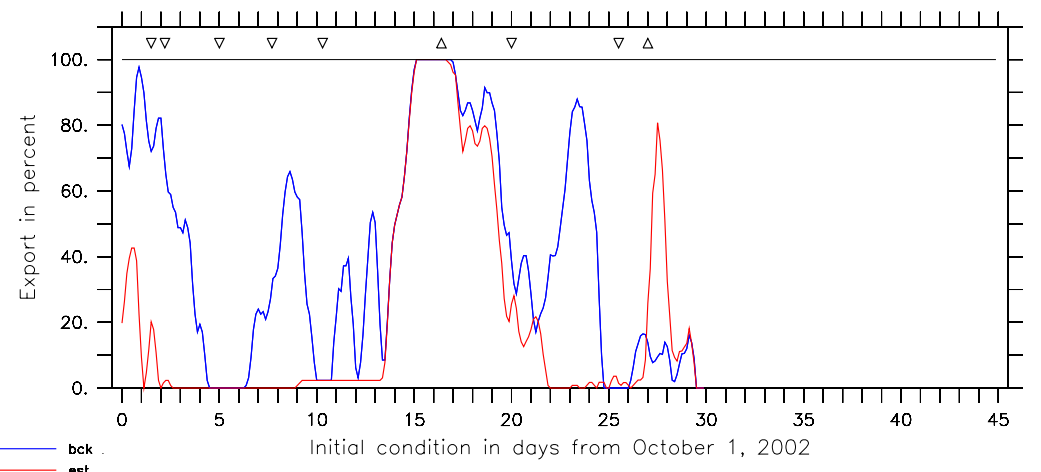

(a)

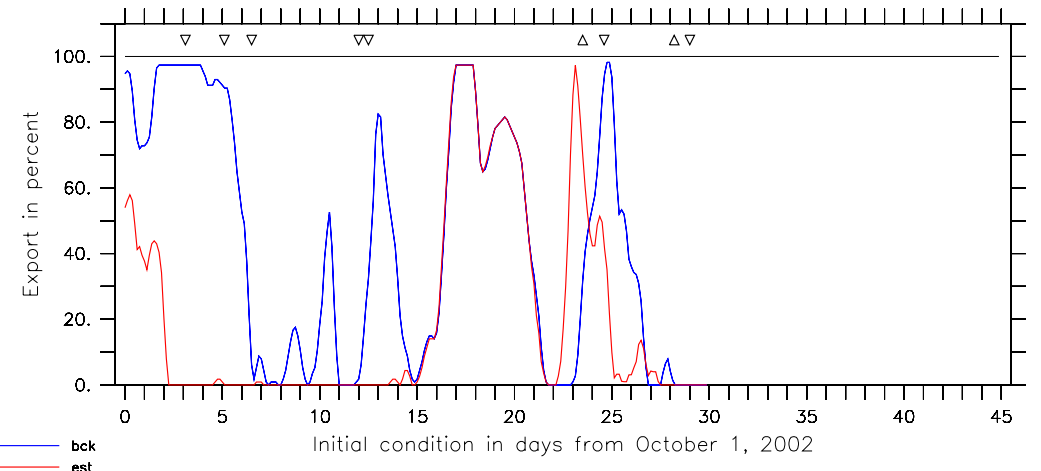

(b)

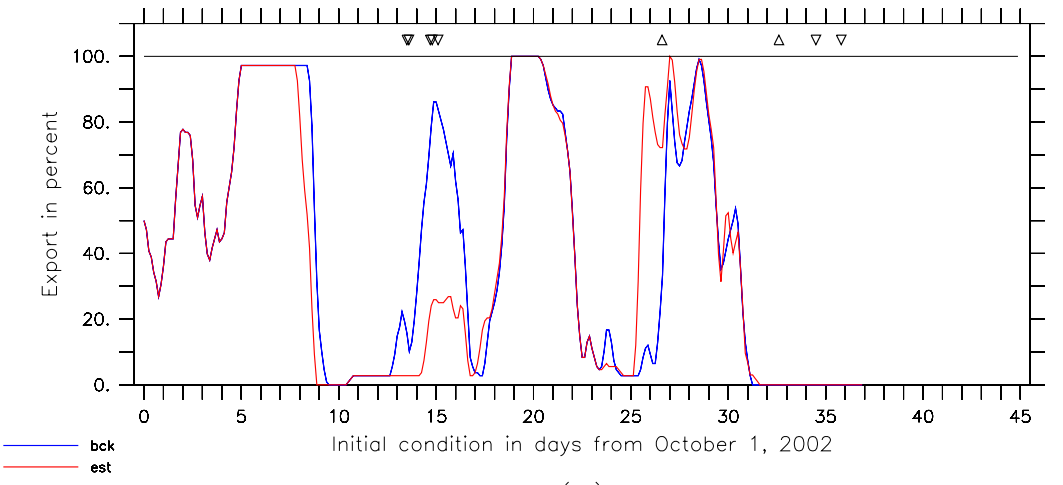

(c)

Figure 9. (a) Export rates, computed from trajectory simulations inside the first guessed velocity fields (blue lines) and the estimated velocity fields (red lines), and fate of the real trajectories (inverted triangle if stays in WAC, triangle if exits from WAC). Plots are represented with respect to the launching time upstream WAC1. (b) Same as Figure 9a for a launching upstream WAC2. (c) Same as Figure 9a for a launching upstream WAC3.

recirculating downstream the cape, while the estimate trajectories show a marked exit point at the tip, in keeping with what shown in the image (Figure 11a).

[52] In summary, the correction appears to significantly improve the description of the boundary current regime capturing its main features when sampled by the drifters.

\section{Summary and Conclusions}

[53] In this paper we present an application of a method for the reconstruction of the velocity field in a coastal flow in the central Adriatic Sea, using data from surface drifters and outputs from the ROMS circulation model. The methodology, previously developed and tested for mesoscale open ocean flows [Taillandier et al., 2006a], has been improved and adapted to the more challenging coastal environment. The presence of strong inhomogeneities, due to the WAC boundary current dynamics over complex bathymetry and coastline, has been accounted for by introducing different space scales $\mathrm{R}$ in the spatial correlation structure of the velocity correction. In practice, two main scales are used, a smaller one for the WAC $(\mathrm{R}=10 \mathrm{~km})$ and a more extended one for the interior flow $(\mathrm{R}=20 \mathrm{~km})$. For these two regimes, anisotropic correlation patterns can be modeled by solutions of a diffusion equation over two distinct domains bounded by their interface, along the WAC offshore edge. 


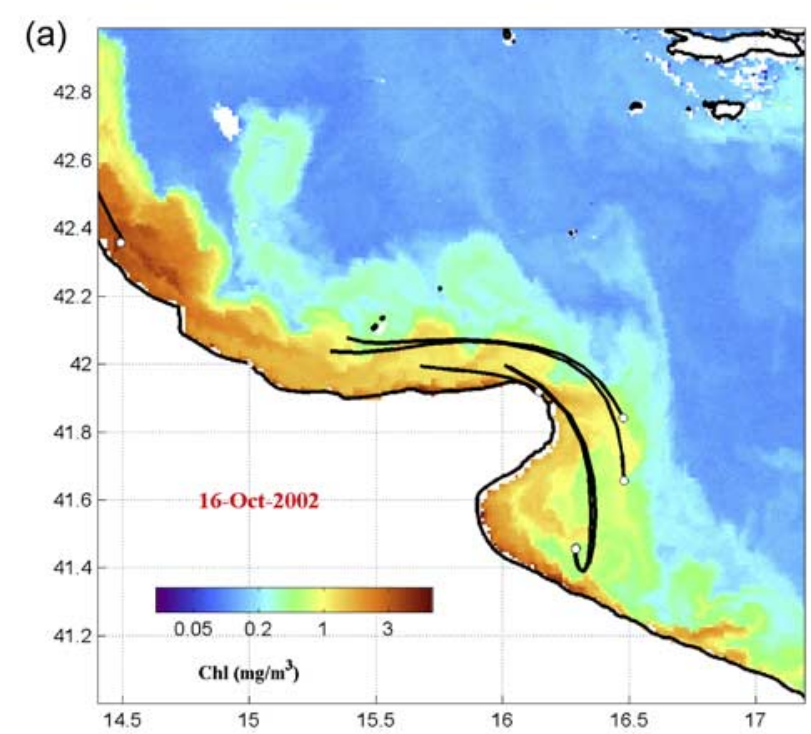

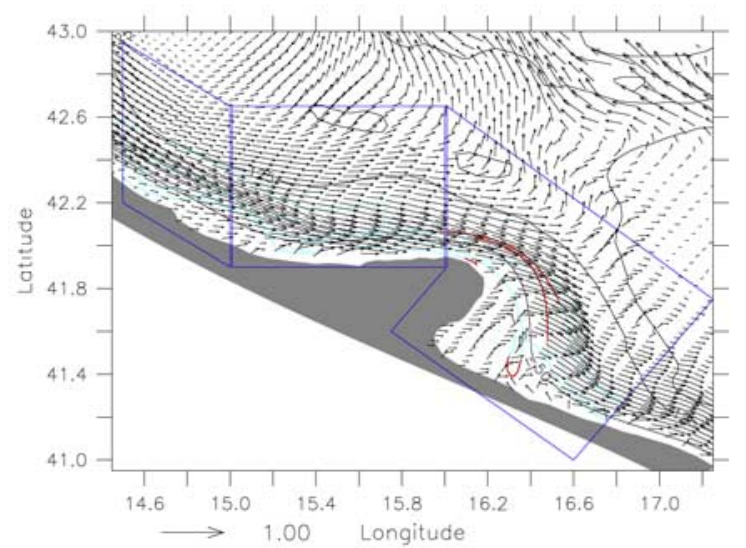

(b)

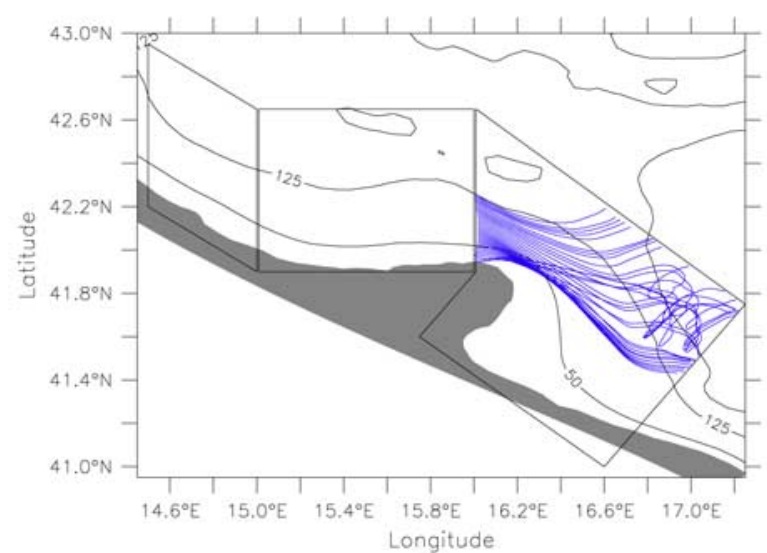

(d)

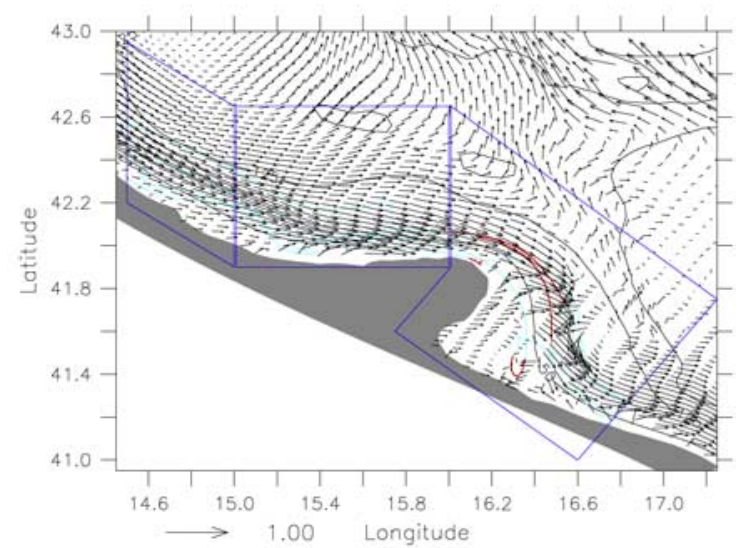

(c)

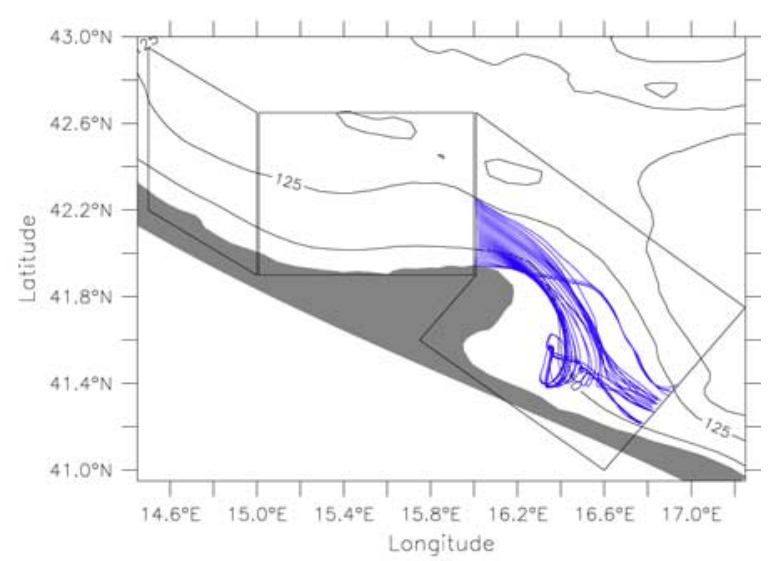

(e)

Figure 10. (a) Sea surface color from MODIS satellite taken at day 16, with current drifter positions in white circles and low-pass filtered drifter track segments the previous $3 \mathrm{~d}$. (b) Mean surface circulation over days 15-16, given by the first guessed velocity fields. (c) Same as Figure 10b for estimated velocity fields. (d) Trajectory simulation inside the first guessed velocity fields for the launching time day 15 . (e) Same as Figure 10d for estimated velocity fields. 


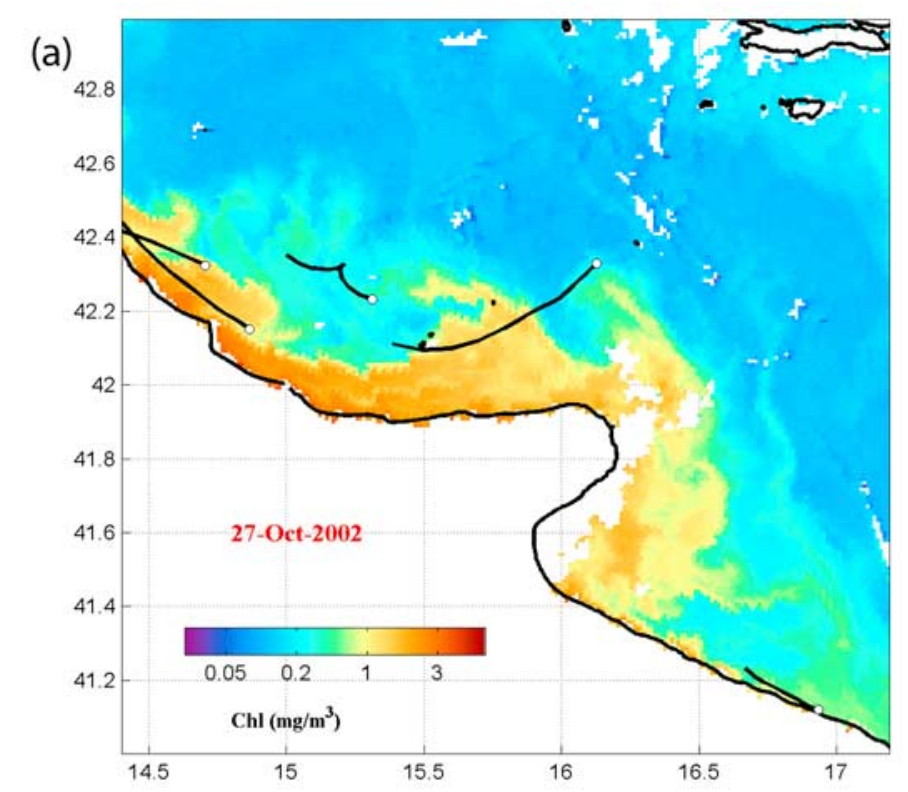

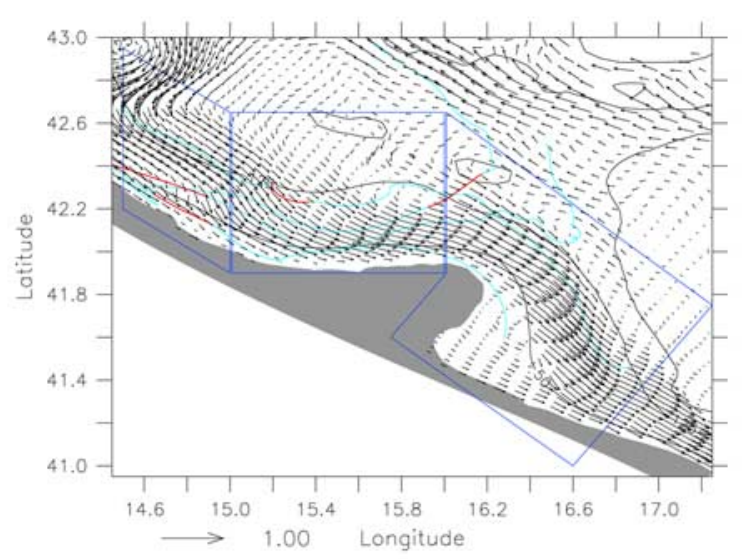

(b)

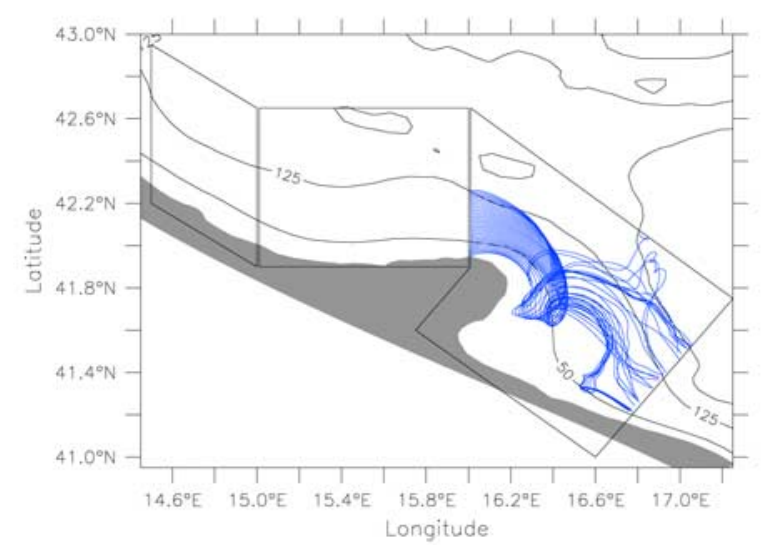

(d)

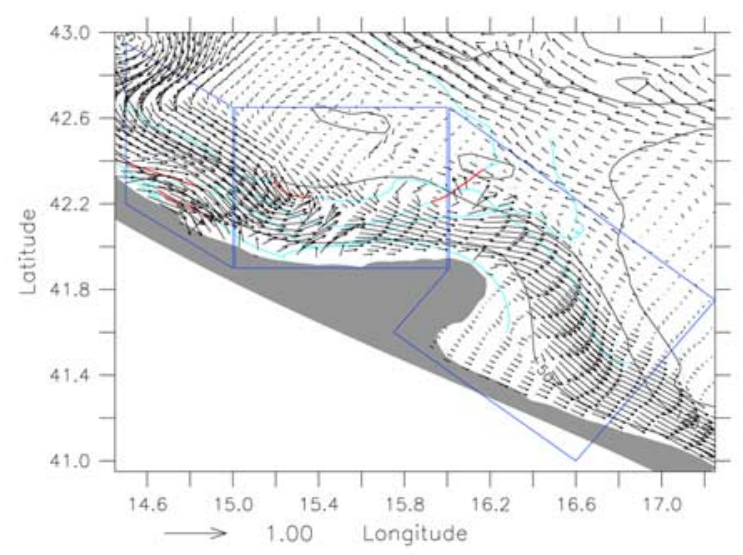

(c)

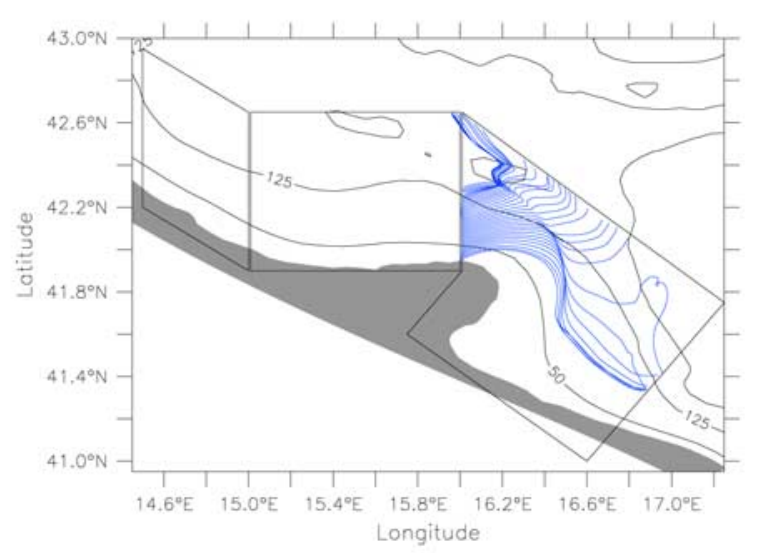

(e)

Figure 11. (a) Sea surface color from MODIS satellite taken at day 27, with current drifter positions in white circles and low-pass-filtered drifter track segments the previous $3 \mathrm{~d}$. (b) Mean surface circulation over days 26-27, given by the first guessed velocity fields. (c) Same as Figure 11b for estimated velocity fields. (d) Trajectory simulation inside the first guessed velocity fields for the launching time day 26. (e) Same as Figure 11d for estimated velocity fields. 
[54] Another challenge found in the coastal flow is that the Eulerian timescales of the velocity field are very small, of the order of $2 \mathrm{~d}$, and comparable to those of the Lagrangian velocity, of the order of $1 \mathrm{~d}$. In this regime, often indicated as "fixed float" approximation [Lumpkin et al., 2002], the fluctuations of the velocity along a particle trajectory are mainly due to the time variability of the advecting Eulerian flow, rather than to its spatial structure as in the "frozen field" approximation. In order to capture the highly varying Eulerian flow, the reconstruction has to be performed over scales close to the Lagrangian timescale so that only few independent position data can be used at each time. As a consequence, the effective data density for each reconstruction is typically low, and it is more difficult to achieve accurate results. In the present application, the velocity is corrected daily considering mesoscale Eulerian flows averaged over $2 \mathrm{~d}$, so that for each reconstruction only 2 independent position data per trajectory are used, given that the Lagrangian timescale is of $1 \mathrm{~d}$.

[55] The velocity reconstruction has been performed for a total of $45 \mathrm{~d}$, obtaining a time series of corrected velocities (estimates). Several diagnostics have been used to evaluate these estimates. In the previous methodological and numerical work of Taillandier et al. [2006a], estimate errors have been quantitatively evaluated using the "twin experiment" approach, where synthetic data are considered and the true state of the ocean is assumed known. Here, since in situ data are used and the real ocean state is not known, the errors cannot be computed directly, and a hierarchy of indirect tests is introduced to evaluate the results. The first set of tests addresses the internal consistency and the impact of the reconstruction, based on the comparison between results from the estimates and results from the "first guessed" fields, i.e., the original ROMS outputs without drifter corrections. The tests include a particle prediction diagnostic and two statistics characterizing the transport in terms of residence times in various regions and export rates from the WAC toward the interior. The results show that the reconstruction is quite effective. The error on the particle prediction is significantly reduced and tends to saturate, showing that the method is internally consistent since the velocity reconstruction is obtained minimizing the differences between observed and simulated trajectories. The residence times and export rates diagnostics appear significantly impacted by the analysis, and indicate an improvement over the first guess with respect to the measured quantities.

[56] The last test is based on a qualitative comparison with data from the MODIS satellite images. The comparison is necessarily qualitative given the intrinsic differences between the drifter information (relative to the upper 1-2 $\mathrm{m}$ of water and filtered for mesoscale reconstruction) and the image information (relative to an instantaneous surface tracer not completely passive). For these reasons, the comparison does not focus on the detailed structure of the field, but rather on the main characteristics of the WAC and its exchange with the interior. The results show that the reconstruction significantly improves the description of the boundary current with respect to the ROMS model first guess, capturing its main features when sampled by the drifters.

[57] In summary, the results of the reconstruction appear very positive and encouraging. Nevertheless there are still some aspects that are not completely resolved and that will have to be addressed in the future. First of all, the reconstruction method is limited by the fact that the velocity correction for each time interval is provided only by the position data occurring during that same interval and acting on a limited area of size $\mathrm{R}$ around them. This is not the case for a complete assimilation procedure, where the information from the data is dynamically propagated by the model, making the correction significantly more powerful. In the future, then, it is desirable that the reconstruction will be part of a more complete assimilation scheme, even though challenges for coastal assimilation are still significant. As an example, while in the open ocean the relationship between velocity and mass field is primarily geostrophic allowing a relatively simple correction balance [e.g., Taillandier et al., 2006 b], in coastal areas the dynamics are expected to be more complex including inertial, bottom friction and direct wind driven components. Studies for enhanced correlation structures in coastal regions are underway, toward multivariate and spatiotemporal descriptions of the background error operators [Echevin et al., 2000; Auclair et al., 2003]. Furthermore, efforts for integrating heterogeneous data (in nature and distribution) would allow provision of such multiscale estimates on the basis of future coastal monitoring networks.

[58] On the other hand, here we focus on the correction of a specific dynamical scale, i.e., the mesoscale with time and space scales of some days and tens kilometers, respectively. Submesoscale motions, including higher frequencies and smaller spatial scales, are expected to play an important role especially for dispersion processes which are of key importance for many practical applications in coastal environment. An important aspect to be addressed in the future then is how to include submesoscale features in the reconstruction, either as actual information on the instantaneous field or as accurate and data-based subgrid-scale parameterization. In order to do that, high-resolution unfiltered data will have to be used, but in general, the realistic drifter density will not be enough to significantly constrain the highresolution field. A possible avenue is to combine data from different sources, for instance, drifters and satellite data. A key element in this process is to improve our understanding of the dynamics in the first upper meters, in terms of air sea interaction and vertical shear distribution, so that surface satellite data can be merged with drifter data in the upper meters. These aspects are also expected to improve the prediction capability of the model itself, in addition to help setting a unified assimilation of satellite and drifter data.

[59] Acknowledgments. Four of the authors are supported by the Office of Naval Research, V.T. and A.G. under grants N00014-05-1-0094 and N00014-05-1-0095, P.M.P. under grant N00014-03-1-0291, and S.C. under grant N00014-05-1-0730 (NICOP, program manager Scott Harper). The MODIS data were downloaded from the Goddard Space Flight Center of the National Aeronautics and Space Administration (NASA). CNR-ISMAR activity was partially supported by P.O.R. "CAINO" (Regione Puglia), VECTOR (Italian MIUR) project, and ECOOP (EU project).

\section{References}

Aref, H. (1984), Stirring by chaotic advection, J. Fluid Mech., 143, 1-21. Auclair, F., P. Marsaleix, and P. De Mey (2003), Space-time structure and dynamics of the forecast error in a coastal circulation model of the Gulf of Lions, Dyn. Atmo. Oceans, 36, 309-346.

Barbanti, R., R. Iungwirth, and P.-M. Poulain, (2005), Stima dell'accuratezza del drifter tipo CODE con GPS nella determinazione della posizione geo- 
grafica, Tech. Rep. 32/2005/OGA/20, Ist. Naz. di Oceanogr. e di Geofis. Sper., Trieste, Italy.

Bignami, F., R. Sciarra, S. Carniel, and R. Santoleri (2007), Variability of Adriatic Sea coastal turbid waters from SeaWiFS imagery, J. Geophys. Res., 112, C03S10, doi:10.1029/2006JC003518.

Budyko, K. (1974), Climate and Life, 508 pp., Academic, San Diego, Calif. Cushman-Roisin, B., and C. E. Naimie (2002), A 3D finite-element model of the Adriatic tides, J. Mar. Syst., 37, 279-297.

Derber, J., and A. Rosati (1989), A global oceanic data assimilation system, J. Phys. Oceanogr., 19, 1333-1347.

Echevin, V., P. De Mey, and G. Evensen (2000), Horizontal and vertical structure of the representer functions for the sea surface measurements in a coastal circulation model, J. Phys. Oceanogr., 30, 2627-2635.

Fairall, C. W., E. F. Bradley, J. E. Hare, A. A. Grachev, and J. B. Edson (2003), Bulk parameterisations of air-sea fluxes: Updates and verification for the COARE algorithm, J. Clim., 16, 571-591.

Hernandez, F., P. Y. Le Traon, and R. A. Morrow (1995), Mapping mesoscale variability of the Azores Current using T/P and ERS-1 altimetry, together with hydrographic and Lagrangian measurements, J. Geophys. Res., 100, $24,995-25,006$

Ide, K., L. Kuznetsov, and C. K. R. T. Jones (2002), Lagrangian data assimilation for point vortex systems, J. Turbulence, 3, 53-59.

Ishikawa, Y. I., T. Awaji, and K. Akimoto (1996), Successive correction of the mean sea surface height by the simultaneous assimilation of drifting buoys and altimetric data, J. Phys. Oceanogr., 26, 2381-2397.

Kamachi, M., and J. O'Brien (1995), Continuous assimilation of drifting buoy trajectory into an equatorial Pacific Ocean model, J. Mar. Syst., 6, $159-178$

Kuznetsov, L., K. Ide, and C. K. R. T. Jones (2003), A method for assimilation of Lagrangian data, Mon. Weather Rev., 131, 2247-2260.

Lumpkin, R., A. M. Treguier, and K. Speer (2002), Lagrangian eddy scales in the northern Atlantic Ocean, J. Phys. Oceanogr., 32, 2440-2452.

Margolin, L., and P. K. Smolarkiewicz (1998), Antidiffusive velocities for multipass donor cell, SIAM J. Sci. Comput., 20, 907-929.

Mauri, E., P.-M. Poulain, and Ž. Južnič-Zonta (2007), MODIS chlorophyll variability in the northern Adriatic Sea and relationship with forcing parameters, J. Geophys. Res., 112, C03S11, doi:10.1029/2006JC003545.

Molcard, A., L. I. Piterbarg, A. Griffa, T. M. Özgökmen, and A. J. Mariano (2003), Assimilation of drifter observations for the reconstruction of the Eulerian circulation field, J. Geophys. Res., 108(C3), 3056, doi:10.1029/ 2001JC001240.

Molcard, A., A. Griffa, and T. Özgökmen (2005), Lagrangian data assimilation in multilayer primitive equation models, J. Atmos. Oceanic Technol. $22,70-83$.

Nodet, M. (2006), Variational assimilation of Lagrangian data in oceanography, Inverse Problems, 22, 245-263.

Poulain, P.-M. (2001), Adriatic Sea surface circulation as derived from the drifter data between 1990 and 1999, J. Mar. Syst., 29, 3-32.

Raicich, F. (1996), On the fresh water balance of the Adriatic Sea, J. Mar Syst., 9, 305-319.
Salman, H., L. Kuznetsov, C. K. R. T. Jones, and K. Ide (2006), A method for assimilating Lagrangian data into a Shallow-water equation ocean model, Mon. Weather Rev., 134, 1081-1101.

Shchepetkin, A. F., and J. C. McWilliams (2003), A method for computing horizontal pressure-gradient force in an oceanic model with a nonaligned vertical coordinate, J. Geophys. Res., 108(C3), 3090, doi:10.1029/ 2001JC001047.

Shchepetkin, A. F., and J. C. McWilliams (2005), The regional ocean modelling system: A split-explicit, free-surface, topography-followingcoordinate oceanic model, Ocean Modell., 9, 347-404.

Steppeler, J., G. Doms, U. Shatter, H. W. Bitzer, A. Gassmann, U. Damrath, and G. Gregoric (2003), Meso-gamma scale forecasts using the nonhydrostatic model LM, Meteorol. Atmos. Phys., 82, 75-96.

Taillandier, V., and A. Griffa (2006), Implementation of position assimilation for ARGO floats in a realistic Mediterranean Sea OPA model and twin experiment testing, Ocean Sci., 2, 223-236.

Taillandier, V., A. Griffa, and A. Molcard (2006a), A variational approach for the reconstruction of regional scale Eulerian velocity fields from Lagrangian data, Ocean Modell., 13, 1-24.

Taillandier, V., A. Griffa, P.-M. Poulain, and K. Béranger (2006), Assimilation of Argo float positions in the northwestern Mediterranean Sea and impact on ocean circulation simulations, Geophys. Res. Lett., 33, L11604, doi:10.1029/2005GL025552.

Ursella, L., P.-M. Poulain, and R. P. Signell (2006), Surface drifter derived circulation in the northern and middle Adriatic Sea: Response to wind regime and season, J. Geophys. Res., 111, C03S04, doi:10.1029/ 2005JC003177 [printed 112 (C3), 2007].

Veneziani, M., A. Griffa, and P. -M. Poulain (2007), Historical drifter data and statistical prediction of particle motion: A case study in the Adriatic Sea, J. Atmos. Oceanic Technol., 24, 235-254.

Warner, J. C., C. R. Sherwood, H. G. Arango, and R. P. Signell (2005), Performance of four turbulence closure models implemented using a generic length scale method, Ocean Modell., 8, 81-113.

Weaver, A. T., and P. Courtier (2001), Correlation modeling on the sphere using a generalized diffusion equation, Q. J. R. Meteorol. Soc., 127, $1815-1846$

S. Carniel and J. Chiggiato, CNR-ISMAR, Castello 1364/A, I-30122 Venice, Italy.

A. Griffa, RSMAS-MPO, 4600 Rickenbacker Causeway, Miami, FL 33149-1098, USA.

P. M. Poulain, INOGS, Borgo Grotta Gigante, 42/c, 34010 Sgonico (Trieste), Italy.

R. Signell, USGS, 384 Woods Hole Road, Woods Hole, MA 02543-1598, USA.

V. Taillandier, LOCEAN, IPSL, Boîte 100-4, place Jussieu, F-75252

Paris Cedex 05, France. (vincent.taillandier@locean-ipsl.upmc.fr) 